\title{
THE MONOTONICITY OF THE ENTROPY FOR A FAMILY OF DEGREE ONE CIRCLE MAPS
}

\author{
LLUÍS ALSEDÀ AND FRANCESC MAÑOSAS
}

\begin{abstract}
For the natural biparametric family of piecewise linear circle maps with two pieces we show that the entropy increases when any of the two parameters increases. We also describe the regions of the parameter space where the monotonicity is strict.
\end{abstract}

\section{Statement of the Results}

In this paper we study the monotonicity of the entropy for a biparametric family of degree one circle maps. The monotonicity of the entropy for particular families of maps of the interval has been considered by several authors for several families (see [MV, BMT, MT, DH]). We consider a problem similar to the one considered in [MV]. We deal with the biparametric family of piecewise linear circle maps with two pieces and we prove that the entropy increases when any of the two slopes increases. We also describe the regions of the parameter space where the monotonicity is strict.

In [AM] a kneading theory for a class of bimodal continuous circle maps of degree one (called class $\mathscr{A}$ ) was developed. The framework of the present study will be that kneading theory. Therefore, this paper has to be considered as a second part of [AM]. Hence, we assume the reader is familiar with the notation, definitions, proofs, and techniques developed in [AM] and we shall use them freely in this paper.

The family we are going to study can be defined as follows (see Figure 1). For $\lambda>1$ and $\mu>0$ we set

$$
G_{\lambda, \mu}(x)= \begin{cases}\lambda x & \text { if } x \in\left[0, \frac{\mu+1}{\mu+\lambda}\right], \\ 1+\mu(1-x) & \text { if } x \in\left[\frac{\mu+1}{\mu+\lambda}, 1\right], \\ E(x)+G_{\lambda, \mu}(D(x)) & \text { if } E(x) \neq 0,\end{cases}
$$

(where $E(\cdot)$ denotes the integer part function and $D(\cdot)$ the decimal part function, i.e., $D(x)=x-E(x))$.

Clearly $G_{\lambda, \mu} \in \mathscr{A}, c_{G_{\lambda, \mu}}=\frac{\mu+1}{\mu+\lambda}$ and $G_{\lambda, \mu}(0)=0$ for all $\lambda>1$ and $\mu>0$. Hence $\underline{I}_{G_{\lambda, \mu}}(0)$ remains constant when the parameters vary. On the other hand,

Received by the editors July 9, 1990.

1980 Mathematics Subject Classification (1985 Revision). Primary 34C35, 54H20.

Key words and phrases. Kneading theory, monotonicity, topological entropy.

This research was supported by DGCICYT grant number PB86-0351. 


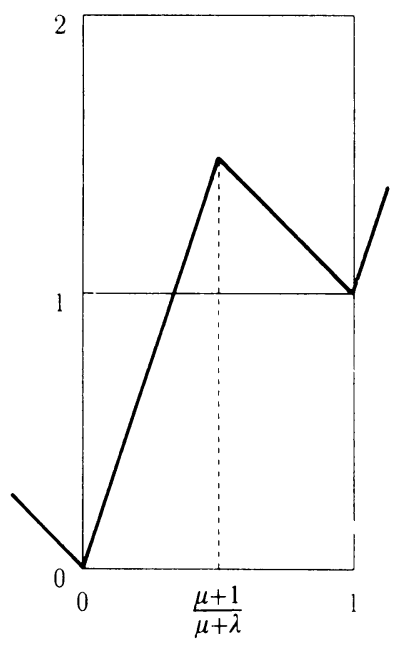

FIGURE 1. The map $G_{3,1}$

for each $\lambda$ and $\mu$ the rotation interval of $G_{\lambda, \mu}$ is of the form $\left[0, b_{\lambda, \mu}\right]$ (of course $\left.b_{\lambda, \mu}>0\right)$.

To simplify the notation we set $c_{\lambda, \mu}=c_{G_{\lambda, \mu}}, K(\lambda, \mu)=\underline{I}_{G_{\lambda, \mu}}\left(c_{\lambda, \mu}\right)$, and $h(\lambda, \mu)=h\left(G_{\lambda, \mu}\right)$. Also we shall write $\left(\lambda^{\prime}, \mu^{\prime}\right) \geq(\lambda, \mu)$ if and only if $\lambda^{\prime} \geq \lambda$ and $\mu^{\prime} \geq \mu$. If additionally either $\lambda^{\prime} \neq \lambda$ or $\mu^{\prime} \neq \mu$ we shall write $\left(\lambda^{\prime}, \mu^{\prime}\right)>$ $(\lambda, \mu)$.

The main results of this paper are the following:

Theorem 1.1. Let $\lambda, \lambda^{\prime}, \mu$, and $\mu^{\prime}$ be such that $(\lambda, \mu)<\left(\lambda^{\prime}, \mu^{\prime}\right)$. Then $K(\lambda, \mu) \leq K\left(\lambda^{\prime}, \mu^{\prime}\right)$. Moreover $K(\lambda, \mu)=K\left(\lambda^{\prime}, \mu^{\prime}\right)$ if and only if for some $m \in \mathbf{Z}, m \geq 1$, we have $b_{\lambda, \mu}=b_{\lambda^{\prime}, \mu^{\prime}}=\frac{1}{m}$ and $\lambda^{\prime m-1} \mu^{\prime}<1$.

Theorem 1.2. Let $\lambda, \lambda^{\prime}, \mu$, and $\mu^{\prime}$ be such that $(\lambda, \mu)<\left(\lambda^{\prime}, \mu^{\prime}\right)$. Then $h(\lambda, \mu) \leq h\left(\lambda^{\prime}, \mu^{\prime}\right)$. Moreover $h(\lambda, \mu)=h\left(\lambda^{\prime}, \mu^{\prime}\right)$ if and only if for some $m \in \mathbf{Z}, m \geq 1$, we have $b_{\lambda, \mu}=b_{\lambda^{\prime}, \mu^{\prime}}=\frac{1}{m}$ and $\mu^{\prime} \leq \lambda^{\prime} /\left(\lambda^{\prime m}-1\right)$. In this last case $h(\lambda, \mu)=h\left(\lambda^{\prime}, \mu^{\prime}\right)=\beta_{0,1 / m}^{-}$.

Remark 1.3. We recall that $\beta_{a, b}^{-}$is the largest root of the equation $R_{a, b}^{-}(z)=\frac{1}{2}$, where $R_{a, b}^{-}(z)=\sum z^{-q}$ and the sum is taken over all pairs $(p, q) \in \mathbf{Z} \times \mathbf{N}$ for which $a<\frac{p}{q}<b$. In the special case in which $a=0$ and $b=\frac{1}{m}$ the formula is specially simple. From Proposition 1.1 of [ALMM] and Theorem C of [ALMS] we get that $\beta_{0,1 / m}^{-}$is the largest root of the polynomial $z^{m+1}-z^{m}-z-1$.

When studying the monotonicity of the entropy (and of the kneading sequences) of a family of piecewise linear maps from $\mathscr{A}$ with two pieces, the more general family to consider is the three parameter family $\Phi_{\lambda, \mu, a}$ defined by $G_{\lambda, \mu}+a$ with $\lambda>1, \mu>0$, and $a \in \mathbf{R}$. As the following example shows, if $a \neq 0$ then we cannot extend Theorem 1.2 to this family. So, in what follows we only consider the family $\Phi_{\lambda, \mu, 0}=G_{\lambda, \mu}$.

Example. Consider $\Phi_{\lambda, \mu, a}$ and $\Phi_{\lambda^{\prime}, \mu^{\prime}, a}$ with $a=0.4, \lambda=2.2, \mu=0.2$, $\lambda^{\prime}=2.5$, and $\mu^{\prime}=0.5$ (see Figure 2 ). 


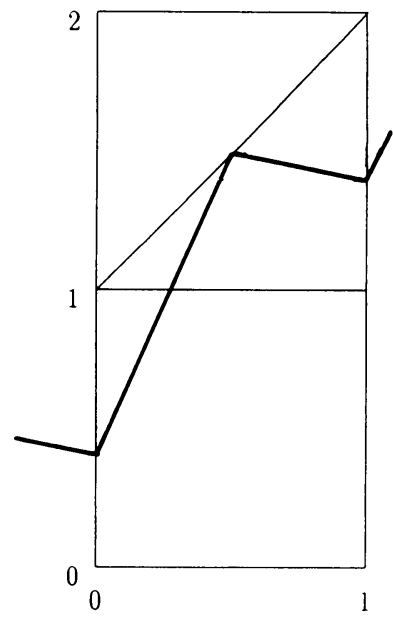

(a)

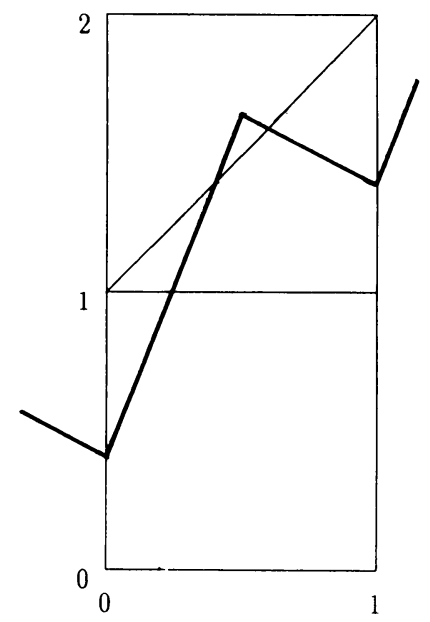

(b)

FIGURE 2. (a) $\Phi_{2.2,0.2,0.4}$, (b) $\Phi_{2.5,0.5,0.4}$

We note that the map $x \rightarrow 0.5-x$ conjugates the map $\Phi_{\lambda, \mu, a}$ with $G_{\lambda, \mu}$. Therefore, from Theorem 1.2 it follows that $h\left(\Phi_{\lambda, \mu, a}\right)>0$. However, $h\left(\Phi_{\lambda^{\prime}, \mu^{\prime}, a}\right)=0$. To see this we can argue as follows. If we denote by $g$ the circle map which has $\Phi_{\lambda^{\prime}, \mu^{\prime}, a}$ as a lifting, we have that $e(0.6)$ and $e(0.4)$ are fixed points of $g$ (where $e(x)=\exp (2 \pi i x)$ denotes the natural projection from $\mathbf{R}$ to $\left.\mathbf{S}^{1}\right)$. Moreover, $e(0.6)$ attracts $e((0.4,1))$ and $e([0,0.4))$ is mapped around the circle only once. Therefore, $g$ has only two nonwandering points which are fixed points and, hence, the entropy of $g$ is zero.

We recall that $\underline{I}_{G_{\lambda, \mu}}(0)$ remains constant when the parameters vary and hence, the problem of the monotonicity of the kneading sequences for the family $G_{\lambda, \mu}$ is essentially one dimensional. However, $\underline{I}_{\Phi_{\lambda, \mu, a}}(0)$ does not remain unchanged when the parameters $\lambda$ and $\mu$ vary and $a \neq 0$. Thus, when considering the case $a \neq 0$ it is necessary to take into account another kneading sequence and the problem becomes two dimensional. In this case the natural extension of Theorem 1.1 in order that we would get $h\left(\Phi_{\lambda^{\prime}, \mu^{\prime}, a}\right)>h\left(\Phi_{\lambda, \mu, a}\right)$ as a corollary would be the following (see Corollary 3.5 of [AM]):

If $\left(\lambda^{\prime}, \mu^{\prime}\right)>(\lambda, \mu)$ then

$$
\underline{I}_{\Phi_{\lambda^{\prime}, \mu^{\prime}, a}}\left(c_{\boldsymbol{\Phi}_{\lambda^{\prime}, \mu^{\prime}, a}}\right) \geq \underline{I}_{\Phi_{\lambda, \mu, a}}\left(c_{\boldsymbol{\Phi}_{\lambda, \mu, a}}\right) \quad \text { and } \quad \underline{I}_{\boldsymbol{\Phi}_{\lambda^{\prime}, \mu^{\prime}, a}}(0) \leq \underline{I}_{\boldsymbol{\Phi}_{\lambda, \mu, a}}(0) \text {. }
$$

For the maps from the example it is not difficult to show that

$$
\underline{I}_{\Phi_{\lambda, \mu, a}}(0)<\underline{I}_{\Phi_{\lambda^{\prime}, \mu^{\prime}, a}}(0)<\underline{I}_{\Phi_{\lambda, \mu, a}}\left(c_{\Phi_{\lambda, \mu, a}}\right)<\underline{I}_{\Phi_{\lambda^{\prime}, \mu^{\prime}, a}}\left(c_{\Phi_{\lambda^{\prime}, \mu^{\prime}, a}}\right)
$$

and the natural extension of Theorem 1.1 does not hold when $a \neq 0$. Thus, in the sequel, we concentrate on the case $a=0$.

Now we start the proofs of Theorems 1.1 and 1.2. The strategy of the proofs for $F_{\mu}$ is as follows. In $\S 2$ we fix $\lambda$ and we study the uniparametric family $F_{\mu}=G_{\lambda, \mu}$. For this family we prove Theorems 2.1 and 2.2 , which are the analogues of Theorems 1.1 and 1.2 , respectively. In $\S 3$ we study the family $F_{\lambda}=G_{\lambda, \mu}$ for $\mu$ fixed. Then we prove Theorems 3.1 and 3.2, which are the analogues for $F_{\lambda}$ of Theorems 1.1 and 1.2, respectively. Then Theorems 1.1 and 1.2 follow from Theorems 2.1, 2.2, 3.1, and 3.2. 


\section{THE FAMILY $F_{\mu}$}

In this section we assume that $\lambda$ is fixed and we study the uniparametric family $F_{\mu}=G_{\lambda, \mu}$. We set $b(\mu)=b_{\lambda, \mu}, h(\mu)=h(\lambda, \mu)$, and $K(\mu)=K(\lambda, \mu)$. The main results of this section are the following versions of Theorems 1.1 and 1.2 for our family $F_{\mu}$ :

Theorem 2.1. Let $\mu_{1}$ and $\mu_{2}$ be such that $\mu_{1}<\mu_{2}$. Then $K\left(\mu_{1}\right) \leq K\left(\mu_{2}\right)$. Moreover $K\left(\mu_{1}\right)=K\left(\mu_{2}\right)$ if and only if there exists $m \in \mathbf{Z}, m \geq 1$ such that $b\left(\mu_{1}\right)=b\left(\mu_{2}\right)=\frac{1}{m}$ and $\lambda^{m-1} \mu_{2}<1$.

Theorem 2.2. Let $\mu_{1}$ and $\mu_{2}$ be such that $\mu_{1}<\mu_{2}$. Then $h\left(\mu_{1}\right) \leq h\left(\mu_{2}\right)$. Moreover $h\left(\mu_{1}\right)=h\left(\mu_{2}\right)$ if and only if there exists $m \in \mathbf{Z}, m \geq 1$ such that $b\left(\mu_{1}\right)=b\left(\mu_{2}\right)=\frac{1}{m}$ and $\mu_{2} \leq \lambda /\left(\lambda^{m}-1\right)$. In this last case $h\left(\mu_{1}\right)=\beta_{0,1 / m}^{-}$.

We introduce some new notation to be used in this section. We set $c_{\mu}=$ $c_{\lambda, \mu}, f_{n}(\mu)=F_{\mu}^{n}\left(c_{\mu}\right)$, and $g_{n}(\mu)=d f_{n}(\mu) / d \mu$ when $D\left(f_{i}(\mu)\right) \notin\left\{0, c_{\mu}\right\}$ for $i=1, \ldots, n-1$. Also set $K(\mu)=A_{0}(\mu) A_{1}(\mu) \cdots={ }^{C} d_{0}(\mu)^{s_{1}(\mu)} d_{1}(\mu) \cdots$, and define

$$
\sigma_{n}(\mu)= \begin{cases}1 & \text { if } \operatorname{Card}\left\{i \in\{1, \ldots, n-1\}: s_{i}(\mu)=R\right\} \text { is even } \\ -1 & \text { if } \operatorname{Card}\left\{i \in\{1, \ldots, n-1\}: s_{i}(\mu)=R\right\} \text { is odd }\end{cases}
$$

Lastly, set $c_{\mu}^{\prime}=d c_{\mu} / d \mu=(\lambda-1) /(\lambda+\mu)^{2}$.

The next result gives a first reduction of our problem to a particular case.

Proposition 2.3. If $\mu^{\prime}>\mu$ and $b\left(\mu^{\prime}\right) \neq b(\mu)$ then $K\left(\mu^{\prime}\right)>K(\mu)$.

Proof. Since $\mu^{\prime}>\mu$ we have $\left(F_{\mu^{\prime}}\right)_{r} \geq\left(F_{\mu}\right)_{r}$ and since $\left(F_{\mu^{\prime}}\right)_{r}$ and $\left(F_{\mu}\right)_{r}$ are nondecreasing we get $\left(F_{\mu}\right)_{r}^{n} \leq\left(F_{\mu^{\prime}}\right)_{r}^{n}$ for all $n \in \mathbf{N}$. Therefore, the rotation number of $\left(F_{\mu}\right)_{r}$ is smaller or equal to the rotation number of $\left(F_{\mu^{\prime}}\right)_{r}$. Then, from Lemma 4.7 of [AM] we obtain $b\left(\mu^{\prime}\right)>b(\mu)$. Therefore, by Theorem B and Lemma 4.9 of [AM] it follows $K\left(\mu^{\prime}\right)>K(\mu)$.

From Proposition 2.3 it follows that it is enough to prove Theorem 2.1 when $b\left(\mu^{\prime}\right)=b(\mu)$.

Now we start the study of the case $b(\mu)=b\left(\mu^{\prime}\right)$. Let $m \in \mathbf{N}$ be such that $m-1<\frac{1}{b(\mu)} \leq m$. Clearly, if $b(\mu) \geq 1$ we have $m=1$ and $b(\mu) \in\left[\frac{1}{m}, \frac{1}{m-1}\right)$ otherwise. We shall keep this assumption until the end of this section. We shall split the study of this case into several lemmas.

We note that when $b(\mu)<1$ (that is $m>1$ ) then $F_{\mu}(x)<1+x$ for all $x \in \mathbf{R}$. Hence $A_{F_{\mu}}(x) \in\left\{{ }^{M} 0,{ }^{L} 0,{ }^{L} 1,{ }^{C} 1,{ }^{R} 1\right\}$ for all $x \in \mathbf{R}$.

Lemma 2.4. The following statements hold.

(1) $\lambda^{m}-\lambda^{m-1}-1>0$;

(2) $\mu \geq 1 /\left(\lambda^{m}-\lambda^{m-1}-1\right)$;

(3) If $m>1$ and $\lambda^{m-1}-\lambda^{m-2}-1>0$ then $\mu<1 /\left(\lambda^{m-1}-\lambda^{m-2}-1\right)$;

(4) If $m>1$ then

$$
\left({ }^{C} 1\left({ }^{L} 0\right)^{m-1}\right)^{\infty}=K\left(\frac{1}{\lambda^{m}-\lambda^{m-1}-1}\right) \leq K(\mu)<\left({ }^{C} 1\left({ }^{L} 0\right)^{m-2}\right)^{\infty} .
$$

Otherwise $K(\mu) \geq\left({ }^{C} 1\right)^{\infty}$. 
Proof. First we prove (4). Assume $m=1$ and suppose that $K(\mu)<\left({ }^{C} 1\right)^{\infty}$. Then we obtain $F_{\mu}\left(c_{\mu}\right)<1+c_{\mu}$ and hence $F_{\mu}(x)<1+x$ for all $x \in \mathbf{R}$; a contradiction because $1 \in L_{F_{\mu}}$.

If $m>1$ we get $b(\mu) \in\left[\frac{1}{m}, \frac{1}{m-1}\right)$. Suppose that $K(\mu)<\left({ }^{C} 1\left({ }^{L} 0\right)^{m-1}\right)^{\infty}$. Then we have $K(\mu)={ }^{C} 1\left({ }^{L} 0\right)^{m-1 L} d \cdots$ (that is $\left.s_{m}(\mu)=L\right)$. Hence $D\left(F_{\mu}^{m}\left(c_{\mu}\right)\right)$ $<c_{\mu}$. On the other hand from Lemma 4.1 of [AM] since $b(\mu) \geq \frac{1}{m}, F_{\mu}$ has a TPO (twist periodic orbit) $P$ of period $m$ and rotation number $\frac{1}{m}$. If $c_{\mu} \in P$, from Lemma 4.4 of [AM] we obtain $K(\mu)=\left({ }^{C} 1\left({ }^{L} 0\right)^{m-1}\right)^{\infty}$. So $c_{\mu} \notin P$. Then, from Lemma 4.4 and Remark 3.1 of $[\mathrm{AM}]$ we get $\left.\underline{I}_{F_{\mu}}\left(\nu_{P}\right)=\left({ }^{L} 1\left({ }^{L} 0\right)^{m-1}\right)\right)^{\infty}$ (recall that $\nu_{P}=\max P \cap[0,1)$ ). Thus, since $\underline{I}_{F_{\mu}}\left(c_{\mu}\right)={ }^{C} 1\left({ }^{L} 0\right)^{m-1} \cdots$ and $\underline{I}_{F_{\mu}}\left(\nu_{P}\right)={ }^{L} 1\left({ }^{L} 0\right)^{m-1} \ldots$ we obtain that $\left.F_{\mu}^{m}\right|_{\left[\nu_{P}, c_{\mu}\right]}$ is linear with slope $\lambda^{m}$ and $\left.E\left(F_{\mu}^{m}\right)\right|_{\left[\nu_{P}, c_{\mu}\right]}=1$. Since $\left(F^{m}-1\right)\left(\nu_{P}\right)=\nu_{P}$ we obtain $\left(F^{m}-1\right)\left(c_{\mu}\right)>c_{\mu}$. Therefore $s_{m}(\mu)=R$; a contradiction.

Now suppose that $\left.K(\mu)>\left({ }^{C} 1\left({ }^{L} 0\right)^{m-2}\right)\right)^{\infty}$. Then $\widehat{\underline{I}}_{F_{\mu}}\left(c_{\mu}^{-}\right)>\left(1^{L}\left(0^{L}\right)^{m-2}\right)^{\infty}$ and from Proposition A of [AM] we get $\left(1^{L}\left(0^{L}\right)^{m-2}\right)^{\infty}$ is a reduced itinerary of $F_{\mu}$ (note that $S^{n}\left(\left(1^{L}\left(0^{L}\right)^{m-2}\right)^{\infty}\right) \leq\left(1^{L}\left(0^{L}\right)^{m-2}\right)^{\infty}$ for all $\left.n \geq 0\right)$. Let $x \in \mathbf{R}$ be such that $\widehat{\underline{I}}_{F_{\mu}}(x)=\left(1^{L}\left(0^{L}\right)^{m-2}\right)^{\infty}$. Then $\rho_{F_{\mu}}(x)=\frac{1}{m-1}$ contradicting the assumption that $b(\mu) \in\left[\frac{1}{m}, \frac{1}{m-1}\right)$.

Now we prove (1) and (2). From the above arguments we know that there exist a TPO $P$ such that $\underline{I}_{F_{\mu}}\left(\nu_{P}\right)$ is either $\left({ }^{L} 1\left({ }^{L} 0\right)^{m-1}\right)^{\infty}$ or $\left({ }^{C} 1\left({ }^{L} 0\right)^{m-1}\right)^{\infty}$. In both cases we get $\lambda^{m-1}\left(\lambda \nu_{P}-1\right)=\nu_{P}$. Therefore $\left(\lambda^{m}-1\right) \nu_{P}=\lambda^{m-1}$. Since $\nu_{P} \leq c_{\mu}<1$ we get $\lambda^{m}-1>\lambda^{m-1}$ and (1) holds. On the other hand we have

$$
\nu_{P}=\frac{\lambda^{m-1}}{\lambda^{m}-1} \leq c_{\mu}=\frac{\mu+1}{\mu+\lambda}
$$

and hence,

$$
\lambda^{m}+\lambda^{m-1} \mu \leq \lambda^{m} \mu-\mu+\lambda^{m}-1
$$

Thus (2) follows.

Lastly we prove (3). Assume that $m>1, \lambda^{m-1}-\lambda^{m-2}-1>0$ and suppose that $\mu \geq 1 /\left(\lambda^{m-1}-\lambda^{m-2}-1\right)$, which is equivalent to

$$
\frac{\lambda^{m-2}}{\lambda^{m-1}-1} \leq \frac{\mu+1}{\mu+\lambda}=c_{\mu}
$$

Set $x=\lambda^{m-2} /\left(\lambda^{m-1}-1\right)$. Clearly $F_{\mu}^{m-1}(x)=1+x$. Hence, $\frac{1}{m-1} \in L_{F_{\mu}} ; \mathrm{a}$ contradiction. This ends the proof of the lemma.

From Lemma 2.4 it follows that we also we may assume that $\lambda^{m}-\lambda^{m-1}-1$ $>0$ and we shall do so in the rest of this section. Also we set $J(m)=$ $\left[1 /\left(\lambda^{m}-\lambda^{m-1}-1\right), \kappa(m)\right)$, where

$$
\kappa(m)= \begin{cases}\frac{1}{\lambda^{m-1}-\lambda^{m-2}-1} & \text { if } m>1 \text { and } \lambda^{m-1}-\lambda^{m-2}-1>0, \\ \infty & \text { if } m=1 \text { or } m>1 \text { and } \lambda^{m-1}-\lambda^{m-2}-1 \leq 0 .\end{cases}
$$

From Lemma 2.4 we can also assume that $\mu \in J(m)$. 
Remark 2.5. From Lemma 2.4(4) it follows that for $m>1$ and for $\mu \in J(m)$ we have $K(\mu)={ }^{C} 1\left({ }^{L} 0\right)^{m-2 L} d \ldots$ with $d \in\{0,1\}$. Therefore, from Proposition A of [AM] we get that if for some $i \geq 0, d_{i}(\mu)=1$ then $s_{i+j}(\mu)=L$ for $j=1, \ldots, m-1$ and $d_{i+j}(\mu)=0$ for $j=1, \ldots, m-2$.

Note that, in general, the map $F_{\mu}$ does not belong to the class $\mathscr{B}$ (see [AM]). The following lemma shows that for some values of the parameter $\mu$ the maps $F_{\mu}$ preserve the properties of the maps of class $\mathscr{B}$. We set $I(m)=\{\mu \in$ $\left.J(m): \mu>\lambda /\left(\lambda^{m}-1\right)\right\}$. We recall that $\mathscr{T}_{F}$ is the set of all preimages of 0 and $c_{F}$ under $F$.

Lemma 2.6. $\mathscr{T}_{F_{\mu}}$ is dense in $\mathbf{R}$ for all $\mu \in I(m)$.

Proof. Suppose that $\mathscr{T}_{F_{\mu}}$ is not dense and let $U$ be the complement of $\operatorname{cl}\left(\mathscr{T}_{F_{\mu}}\right)$. The set $U$ is open and hence it is a countable union of disjoint open intervals. Let $U^{\prime}=U \cap[0,1)$ (note that $U=\bigcup_{m \in \mathbf{Z}} m+U^{\prime}$ ). Number the intervals of $U^{\prime}$ by $\left\{U_{k}\right\}_{k=1}^{\infty}$ and denote by $c_{k}$ the length of the $k$ th interval. Clearly $\sum_{k=0}^{\infty} c_{k} \leq 1$. Hence $\lim _{k \rightarrow \infty} c_{k}=0$ and there exists $k_{0} \in \mathbf{N}$ such that $c_{k} \leq c_{k_{0}}$ for every $k \in \mathbf{N}$. Note that $U^{\prime} \cap\left\{0, c_{\mu}\right\}=\varnothing$. Therefore either $U_{k_{0}} \subset\left(0, c_{\mu}\right)$ or $U_{k_{0}} \subset\left(c_{\mu}, 1\right)$. In the first case we get $F_{\mu}^{\prime}(x)=\lambda>1$ for all $x \in U_{k_{0}}$. In the second case, from Remark 2.5 we obtain $\left(F_{\mu}^{m}\right)^{\prime}(x)=\lambda^{m-1} \mu>\lambda^{m} /\left(\lambda^{m}-1\right)>1$ for all $x \in U^{\prime}$. Since $F(U) \subset U$ we have that either $F$ or $F^{m}$ maps $U_{k_{0}}$ to a larger interval of $U$; a contradiction.

Remark 2.7. From the above lemma it is easy to see that all of the results proved in [AM] for class $\mathscr{B}$ (that is from Lemma 3.6 until Proposition 3.12) are also valid for the maps $F_{\mu}$ when $\mu \in I(m)$.

Now we are interested in characterizing the values of the parameter $\mu$ for which there exist a subinterval of $[0,1]$ containing $c_{\mu}$ which is invariant for $F^{m}-1$. This problem is closely related to the characterization of the values $\lambda$ for which $\lambda /\left(\lambda^{m}-1\right) \in J(m)$. To this end we introduce the following family of polynomials. Set

$$
P_{m}(\lambda)=\lambda^{m+1}-2 \lambda^{m}-\lambda+1 .
$$

Lemma 2.8. For each $m \geq 1$ the polynomial $P_{m}(\lambda)$ has a unique root larger than one. Moreover if we denote this root by $\pi_{m}$ we have

(1) $2<\pi_{m}<3$;

(2) If $n<m$ then $\pi_{n}>\pi_{m}$;

(3) $\pi_{m}^{m}-\pi_{m}^{m-1}-1>0$.

Proof. Since $P_{m}(2)=-1$ for all $m \geq 1$, the equation $P_{m}(\lambda)=0$ is equivalent to the equation $\lambda^{m}=R(\lambda)$ with $R(\lambda)=\frac{\lambda-1}{\lambda-2}$.

Since $\lim _{\lambda \downarrow 2} R(\lambda)=\infty, R(3)=2$, and $\left.R\right|_{(2, \infty)}$ is decreasing we obtain (1) and (2) (see Figure 3).

Since $\pi_{m}^{m}=R\left(\pi_{m}\right)$ we get

$$
\pi_{m}^{m}-\pi_{m}^{m-1}-1=\frac{\pi_{m}-1}{\pi_{m}-2}-\frac{\pi_{m}-1}{\left(\pi_{m}-2\right) \pi_{m}}-1=\frac{\left(\pi_{m}-1\right)^{2}}{\pi_{m}\left(\pi_{m}-2\right)}-1 .
$$

Since $\pi_{m}>2$ we obtain (3).

It is not difficult to show that for $\mu \in J(m)$ the point $x=\lambda^{m-1} /\left(\lambda^{m}-1\right)$ is the largest point in $[0,1)$ of a TPO of period $m$ and rotation number $\frac{1}{m}$ (in fact $\left.\underline{I}_{F_{\mu}}(x)=\left({ }^{L} 1\left({ }^{L} 0\right)^{m-1}\right)^{\infty}\right)$. Let $x_{\mu}$ be the $F_{\mu}$-conjugate of $x$. Clearly, the 


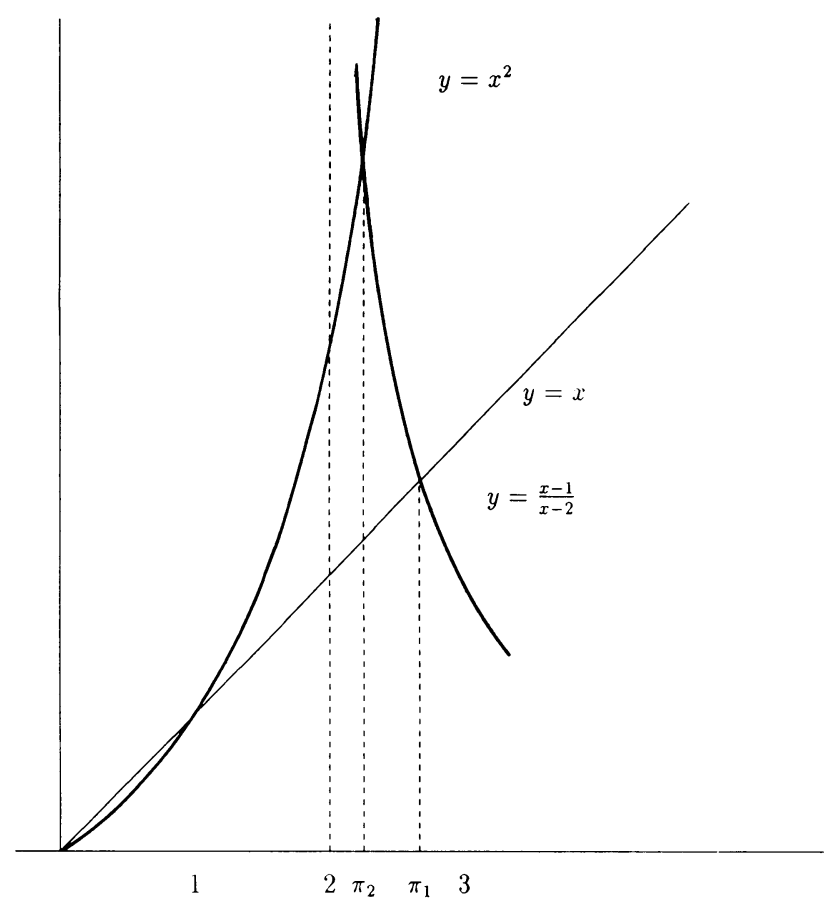

Figure 3. The roots $\pi_{m}$

existence of a subinterval of $[0,1]$ containing $c_{\mu}$ and invariant under $D \circ F^{m}$ is equivalent to the fact that $D\left(F^{m}\left(c_{\mu}\right)\right) \leq x_{\mu}$. In the next lemma we relate this problem to the fact that $\lambda /\left(\lambda^{m}-1\right) \in J(m)$. Also we characterize the situation $\lambda /\left(\lambda^{m}-1\right) \in J(m)$ in terms of the roots of $P_{m}(\lambda)$ and we obtain some preliminary results about kneading sequences. For this we shall use the following sequence

$$
\underline{B}={ }^{C} 1\left({ }^{L} 0\right)^{m-1 R} 1\left(\left({ }^{L} 0\right)^{m-1 L} 1\right)^{\infty} .
$$

Lemma 2.9. The following statements hold,

(1) $\lambda /\left(\lambda^{m}-1\right) \in J(m)$ if and only if $\lambda \geq \pi_{m}$;

(2) For $\mu \in J(m), f_{m}(\mu) \leq 1+x_{\mu}$ if and only if $\mu \leq \lambda /\left(\lambda^{m}-1\right)$;

(3) If $\lambda \geq \pi_{m}$ and $\mu, \mu^{\prime} \in J(m)$ with $\mu<\lambda /\left(\lambda^{m}-1\right)<\mu^{\prime}$ then

$$
K(\mu) \leq K\left(\frac{\lambda}{\lambda^{m}-1}\right)=\underline{B}<K\left(\mu^{\prime}\right) ;
$$

(4) If $\lambda<\pi_{m}$ and $\mu \in J(m)$ then $K(\mu)>\underline{B}$.

Proof. (1) By Lemma 2.8 the equation $\lambda \geq \pi_{m}$ is equivalent to the equation $P_{m}(\lambda) \geq 0$ and this is equivalent to $\lambda /\left(\lambda^{m}-1\right) \geq 1 /\left(\lambda^{m}-\lambda^{m-1}-1\right)$. To see that $\lambda /\left(\lambda^{m}-1\right) \in J(m)$ when $\lambda \geq \pi_{m}$ it only remains to show that $\lambda /\left(\lambda^{m}-1\right)<$ $\kappa(m)$. If $\kappa(m)=\infty$ there is nothing to prove. Now assume that $m>1$ and $\lambda^{m-1}-\lambda^{m-2}-1>0$. Then we have

$$
\frac{\lambda}{\lambda^{m}-1}=\frac{1}{\lambda^{m-1}-1 / \lambda}<\frac{1}{\lambda^{m-1}-\lambda^{m-2}-1}=\kappa(m)
$$

and (1) holds. 
(2) First we compute $x_{\mu}$. We recall that $x_{\mu}$ is the $F_{\mu}$-conjugate of $x=$ $\lambda^{m-1} /\left(\lambda^{m}-1\right)$. Then we have

$$
\mu\left(1-x_{\mu}\right)+1=F_{\mu}\left(x_{\mu}\right)=F_{\mu}(x)=\lambda \frac{\lambda^{m-1}}{\lambda^{m}-1} .
$$

Hence,

$$
x_{\mu}=1-\frac{1}{\mu\left(\lambda^{m}-1\right)} .
$$

Now we consider the inequality

$$
f_{m}(\mu)=\lambda^{m-1}\left(\lambda c_{\mu}-1\right)+1>2-\frac{1}{\mu\left(\lambda^{m}-1\right)}=1+x_{\mu} .
$$

An easy computation shows that for $\mu \in J(m)$ the above inequality is equivalent to

$$
\left(\mu-\frac{\lambda}{\lambda^{m}-1}\right)\left(\mu-\frac{1}{\lambda^{m}-\lambda^{m-1}-1}\right)>0 .
$$

Then, for all $\mu \in J(m) \quad(*)$ holds if and only if $\mu>\lambda /\left(\lambda^{m}-1\right)$. This proves (2).

Now we assume that $\lambda \geq \pi_{m}$ and we compute $K\left(\lambda /\left(\lambda^{m}-1\right)\right)$. We get

$$
c_{\lambda /\left(\lambda^{m}-1\right)}=\frac{\lambda /\left(\lambda^{m}-1\right)+1}{\lambda /\left(\lambda^{m}-1\right)+\lambda}=\frac{\lambda^{m}+\lambda-1}{\lambda^{m+1}} \quad \text { and } \quad x_{\lambda /\left(\lambda^{m}-1\right)}=1-\frac{1}{\lambda}=\frac{\lambda-1}{\lambda} \text {. }
$$

Therefore

$$
f_{m}\left(\frac{\lambda}{\lambda^{m}-1}\right)=\lambda^{m-1}\left(\lambda\left(\frac{\lambda^{m}+\lambda-1}{\lambda^{m+1}}\right)-1\right)=1+x_{\lambda /\left(\lambda^{m}-1\right)} .
$$

Hence,

$$
K\left(\frac{\lambda}{\lambda^{m}-1}\right)={ }^{C} 1\left({ }^{L} 0\right)^{m-1} \underline{I}_{F_{\left.\lambda / \lambda^{m}-1\right)}}\left(x_{\lambda /\left(\lambda^{m}-1\right)}\right)={ }^{C} 1\left({ }^{L} 0\right)^{m-1 R} 1\left(\left({ }^{L} 0\right)^{m-1 L} 1\right)^{\infty}=\underline{B} .
$$

Let $\mu \in\left(1 /\left(\lambda^{m}-\lambda^{m-1}-1\right), \lambda /\left(\lambda^{m}-1\right)\right)$. Then, since $f_{m}(\mu)<1+x_{\mu}<2$ we have

$$
K(\mu)={ }^{C} 1\left({ }^{L} 0\right)^{m-1} \underline{I}_{F_{\mu}}\left(f_{m}(\mu)\right) \leq{ }^{C} 1\left({ }^{L} 0\right)^{m-1} \underline{I}_{F_{\mu}}\left(x_{\mu}\right)=\underline{B} .
$$

If $\mu \in\left(\lambda /\left(\lambda^{m}-1\right), \kappa(m)\right)$, from (2) we obtain $f_{m}(\mu)>1+x_{\mu}$. If $f_{m}(\mu) \geq 2$, then

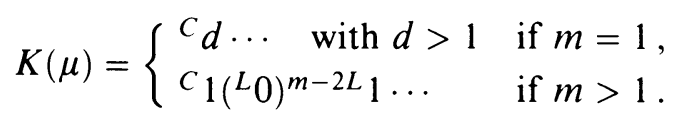

Thus, in both cases we have $K(\mu)>\underline{B}$. If $f_{m}(\mu)<2$ we have $K(\mu)=$ ${ }^{C} 1\left({ }^{L} 0\right)^{m-1} \underline{I}_{F_{\mu}}\left(f_{m}(\mu)\right)$.

On the other hand, since $1+x_{\mu}<f_{m}(\mu)<2$, we get $x_{\mu}<D\left(f_{m}(\mu)\right)$ and from Lemma 3.7 of $[\mathrm{AM}]$ and Remark 2.7 we have $\underline{I}_{F_{\mu}}\left(f_{m}(\mu)\right)>\underline{I}_{F_{\mu}}\left(x_{\mu}\right)$. So $K(\mu)>\underline{B}$.

(4) When $\lambda<\pi_{m}$, from (2), we get $f_{m}(\mu)>1+x_{\mu}$ for all $\mu \in J(m)$. Then, by the same arguments as in the proof of (3) we obtain $K(\mu)>\underline{B}$.

Remark 2.10. From Lemma 2.9 it follows that $f_{m}(\mu)<1+x_{\mu}$ for $\lambda>\pi_{m}$ and for $\mu \in\left(1 /\left(\lambda^{m}-\lambda^{m-1}-1\right), \lambda /\left(\lambda^{m}-1\right)\right)$. Since $F_{\mu}^{m}\left(\lambda^{m-1} /\left(\lambda^{m}-1\right)\right)=$ $1+\lambda^{m-1} /\left(\lambda^{m}-1\right), F_{\mu}^{m}\left(c_{\mu}\right)=f_{m}(\mu)<1+x_{\mu}$, and $F_{\mu}^{m}\left(x_{\mu}\right)=1+\lambda^{m-1} /\left(\lambda^{m}-1\right)$ 


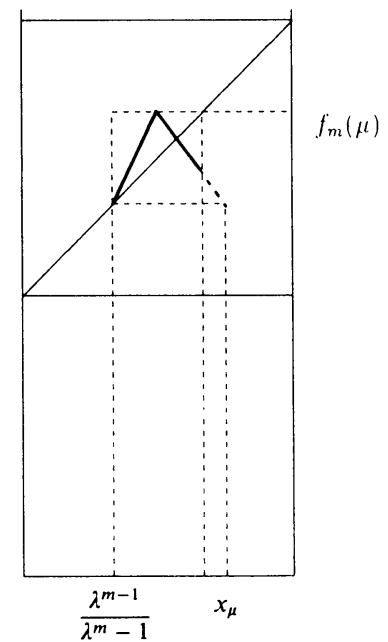

FIGURE 4. The map $\left.\left(F_{\mu}^{m}-1\right)\right|_{\left[\lambda^{m-1} /\left(\lambda^{m}-1\right), f_{m}(\mu)-1\right]}$

we get that $F_{\mu}^{m}$ maps the interval $\left[\lambda^{m-1} /\left(\lambda^{m}-1\right), f_{m}(\mu)-1\right]$ into itself (see Figure 4). On the other hand, since

$$
\underline{I}_{F_{\mu}}(y)={ }^{L} 1\left({ }^{L} 0\right)^{m-1} \cdots \text { for all } y \in\left(\lambda^{m-1} /\left(\lambda^{m}-1\right), c_{\mu}\right)
$$

and

$$
\underline{I}_{F_{\mu}}(z)={ }^{R} 1\left({ }^{L} 0\right)^{m-1} \cdots \quad \text { for all } z \in\left(c_{\mu}, f_{m}(\mu)-1\right)
$$

we get that $\left.F_{\mu}^{m}\right|_{\left[\lambda^{m-1} /\left(\lambda^{m}-1\right), c_{\mu}\right]}$ has slope $\lambda^{m}$ and $\left.F_{\mu}^{m}\right|_{\left[c_{\mu}, f_{m}(\mu)-1\right]}$ has slope $-\lambda^{m-1} \mu$.

In view of Lemma 2.9 we can split our problem into two different cases. The first one is $\lambda>\pi_{m}$ and $\mu \in\left[1 /\left(\lambda^{m}-\lambda^{m-1}-1\right), \lambda /\left(\lambda^{m}-1\right)\right]$ and the second one is $\mu \in\left(\max \left(1 /\left(\lambda^{m}-\lambda^{m-1}-1\right), \lambda /\left(\lambda^{m}-1\right)\right), \kappa(m)\right)$. To study the monotonicity of $K(\mu)$ in the first case we use a result of Misiurewicz and Visinescu (see [MV]) about maps of the interval. To state it we introduce new notation.

For $\lambda>1$ and $\mu>0$ we define $H_{\lambda, \mu}:[0,1] \rightarrow[0,1]$ as follows (see Figure 5 on next page)

$$
H_{\lambda, \mu}(x)= \begin{cases}\lambda x & \text { if } x \in\left[0, \frac{1}{\lambda}\right], \\ -\mu\left(x-\frac{1}{\lambda}\right)+1 & \text { if } x \in\left[\frac{1}{\lambda}, 1\right] .\end{cases}
$$

In order that $H_{\lambda, \mu}$ maps $[0,1]$ into itself it is necessary that $-\mu\left(x-\frac{1}{\lambda}\right)+1 \geq$ 0 . Thus we obtain $\mu \leq \frac{\lambda}{\lambda-1}$ or equivalently,

$$
\frac{1}{\lambda}+\frac{1}{\mu} \geq 1
$$

Remark 2.11. Consider the family $\widetilde{H}_{\lambda, \mu}$ defined as follows:

$$
\tilde{H}_{\lambda, \mu}(x)= \begin{cases}\lambda x & \text { if } x \in[0, c], \\ -\mu(x-c)+\lambda c & \text { if } x \in[c, 1] .\end{cases}
$$

Note that $\tilde{H}_{\lambda, \mu}$ maps $[0,1]$ into itself if and only if $\lambda c \leq 1$. By the above considerations we get that $\widetilde{H}_{\lambda, \mu}$ maps $[0,1]$ into itself if and only if $\frac{1}{\lambda}+\frac{1}{\mu} \geq 1$ (or equivalently $\mu \leq \frac{\lambda}{\lambda-1}$ ). 


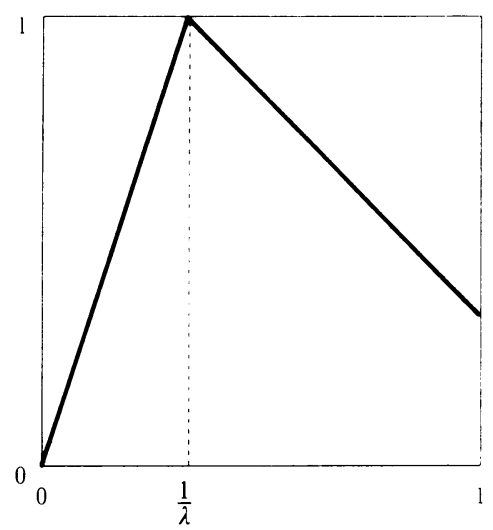

Figure 5. The map $H_{3,1}$

The maps $H_{\lambda, \mu}$ with $\lambda>1$ and $\mu \leq \frac{\lambda}{\lambda-1}$ are called skew tent maps in [MV] and they form a biparametric family of unimodal maps of the interval with a local maximum $d_{\lambda, \mu}=d_{\lambda}=\frac{1}{\lambda}$. Set $\widetilde{K}(\lambda, \mu)=\underline{I}_{H_{\lambda, \mu}}\left(d_{\lambda, \mu}\right)$, where the notion of itinerary is the usual in unimodal maps (i.e., $A(x)$ is $L, C$, or $R$ according as $x$ is less, equal, or greater than $d_{\lambda, \mu}=\frac{1}{\lambda}$ ). The following theorem studies the monotonicity of the kneading sequences of the skew tent maps.

Theorem 2.12. If $(\lambda, \mu)<\left(\lambda^{\prime}, \mu^{\prime}\right)$ then $\widetilde{K}(\lambda, \mu) \leq \widetilde{K}\left(\lambda^{\prime}, \mu^{\prime}\right)$. Moreover if $\mu^{\prime}>1$ then $K(\lambda, \mu)<K\left(\lambda^{\prime}, \mu^{\prime}\right)$.

Theorem 2.12 has been proved by Misiurewicz and Visinescu in the case $\mu>1$ (see [MV]). However, the extension to the case $\mu \leq 1$ is trivial.

Proposition 2.13. Let $\lambda, \mu_{1}$ and $\mu_{2}$ be such that $\lambda>\pi_{m}$ and

$$
1 /\left(\lambda^{m}-\lambda^{m-1}-1\right) \leq \mu_{1}<\mu_{2} \leq \lambda /\left(\lambda^{m}-1\right) \text {. }
$$

Then $K\left(\mu_{1}\right) \leq K\left(\mu_{2}\right)$. Moreover if $\lambda^{m-1} \mu_{2}>1$ then $K\left(\mu_{1}\right)<K\left(\mu_{2}\right)$.

Proof. By Remark 2.10 we get that $F_{\mu}^{m}-1$ maps the interval $\left[\lambda^{m-1} /\left(\lambda^{m}-1\right)\right.$, $\left.f_{m}(\mu)-1\right]$ into itself (see Figure 4) and $\left.\left(F_{\mu}^{m}-1\right)\right|_{\left[\lambda^{m-1} /\left(\lambda^{m}-1\right), f_{m}(\mu)-1\right]}$ is a rescaled version of $H_{\lambda^{m}, \lambda^{m-1} \mu}$. Also we note that $K(\mu)$ is directly obtained from $\widetilde{K}\left(\lambda^{m}, \lambda^{m-1} \mu\right)$ by substituting the symbols $L, C$, and $R$ by ${ }^{L} 1\left({ }^{L} 0\right)^{m-1}$, ${ }^{C} 1\left({ }^{L} 0\right)^{m-1}$, and ${ }^{R} 1\left({ }^{L} 0\right)^{m-1}$ respectively. Hence $K\left(\mu_{1}\right) \leq K\left(\mu_{2}\right)$ if and only if $\widetilde{K}\left(\lambda^{m}, \lambda^{m-1} \mu_{1}\right) \leq \widetilde{K}\left(\lambda^{m}, \lambda^{m-1} \mu_{2}\right)$. Then the proposition follows from Theorem 2.12 .

In what follows we consider the second case. That is

$$
\mu \in I(m)=\left(\max \left(1 /\left(\lambda^{m}-\lambda^{m-1}-1\right), \lambda /\left(\lambda^{m}-1\right)\right), \kappa(m)\right) .
$$

We study this case through a sequence of lemmas. To do this we need again some more definitions. Each sequence of the form ${ }^{s_{0}} d_{0}^{s_{1}} d_{1} \ldots s_{n-1} d_{n-1}$ with $s_{i} \in\{M, L, C, R\}$ and $d_{i} \in \mathbf{Z}$ for all $i=0, \ldots, n-1$ will be called and $n$ strip. Let $\underline{A}^{n}$ be an $n$-strip. Then $\sigma\left(\underline{A}^{n}\right)$ denotes the parity of $\left(s_{0}, \ldots, s_{n-1}\right)$. That is $\sigma\left(\underline{A}^{n}\right)$ will be +1 or -1 according as $\left(s_{0}, \ldots, s_{n-1}\right)$ has an even or odd number of symbols $R$. Also we define $\Delta_{A^{n}}$ as the interior of the set of $\mu$ 's such that $K(\mu)$ starts with $\underline{A}^{n}$. We note that if $\Delta_{A^{n}} \neq \varnothing$ then $s_{0}=C$ and $\left.f_{n}\right|_{\Delta_{A^{n}}}$ is a rational function on $\mu$. Now set $\Gamma_{\underline{A}^{n}}=\Delta_{\underline{A}^{n}} \cap I(m)$. 


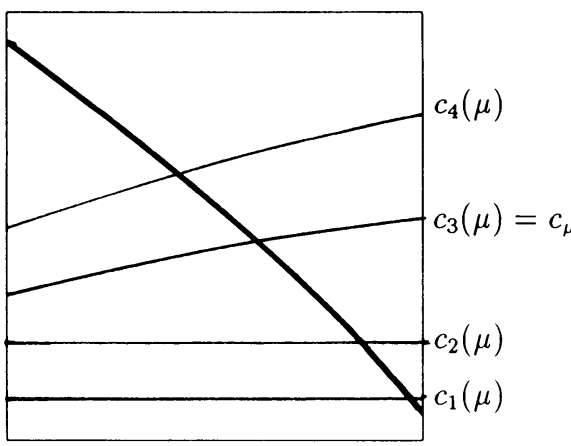

(a)

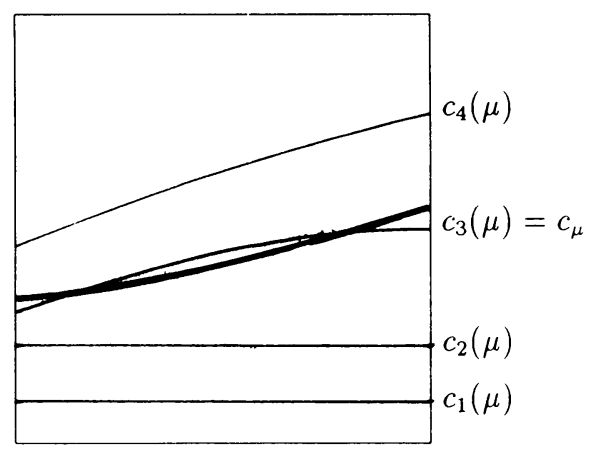

(b)

Figure 6. (a) shows a decreasing map. Hence it is strongly decreasing. (b) shows an increasing map which is not strongly increasing. In both pictures the curves $c_{i}(\mu)$ are the graphs of the turning points depending on $\mu$

Let $(a, b) \subset \mathbf{R}^{+}$and $f:(a, b) \rightarrow[0,1]$ be a continuous map. We say that $f$ is strongly increasing if $f$ is increasing and for all $\mu, \mu^{\prime} \in(a, b)$ with $\mu<\mu^{\prime}$ we have $A_{\mu}(f(\mu)) \leq A_{\mu^{\prime}}\left(f\left(\mu^{\prime}\right)\right) \quad\left(A_{\mu}(x)\right.$ denotes the $F_{\mu}$-address of $\left.x\right)$. We say that $f$ is strongly decreasing if $f$ is decreasing and for all $\mu, \mu^{\prime} \in(a, b)$ with $\mu<\mu^{\prime}$ we have $A_{\mu}(f(\mu)) \geq A_{\mu^{\prime}}\left(f\left(\mu^{\prime}\right)\right)$. Note that since each turning point of $F_{\mu}$ depend on $\mu$ in a nondecreasing way, to show that $f$ is strongly decreasing it is enough to show that $f$ is decreasing (see Figure 6(a)). We also note that the notions of strongly increasing and increasing are not equivalent (see Figure 6(b)).

Remark 2.14. The situation described in Figure 6(b) is not possible if $f^{\prime}(\mu)-$ $c_{k}^{\prime}(\mu)>0$ for all $\mu$.

The strategy of the proof of Theorem 2.1 in this case is as follows. We shall show by induction that for all $n>0$ and for each $n$-strip $\underline{A}^{n}$ such that $\Gamma_{\underline{A}^{n}} \neq \varnothing$ the set $\Gamma_{\underline{A}^{n}}$ is an interval in which $\left.f_{n}\right|_{\Gamma_{A^{n}}}$ is strongly increasing or strongly decreasing according to the parity of $\underline{A}^{n}$. From this it follows easily the monotonicity of the kneading sequence depending on $\mu$. Unfortunately to be able to make the induction step we shall need to prove some additional properties. We start this proof with some preliminary lemmas. See Figure 7 (next page) to illustrate the first one of them.

Lemma 2.15. Let $f, g:[a, b) \rightarrow \mathbf{R}$ with $b \in \mathbf{R} \cup\{\infty\}$ be functions such that $f(a)<g(a)$ and $f$ and $g$ are convex and concave on $[a, b)$, respectively. Then the equation $f(x)=g(x)$ has at most one solution.

Proof. Assume that there exist $z$ and $y$ such that $a<z<y, f(z)=g(z)$, and $f(y) \leq g(y)$. Then $z=t a+(1-t) y$ for some $t \in(0,1)$ and we get $f(z) \leq t f(a)+(1-t) f(y)<t g(a)+(1-t) g(y) \leq g(z) ;$ a contradiction.

Remark 2.16. From Lemma 2.15 it follows that if $f:[a, b) \rightarrow \mathbf{R}$ is a differentiable function, increasing and convex and $f(a)<c_{a}$ then $f$ is strongly increasing. Moreover, in this situation, to see that $f$ is strongly increasing it 


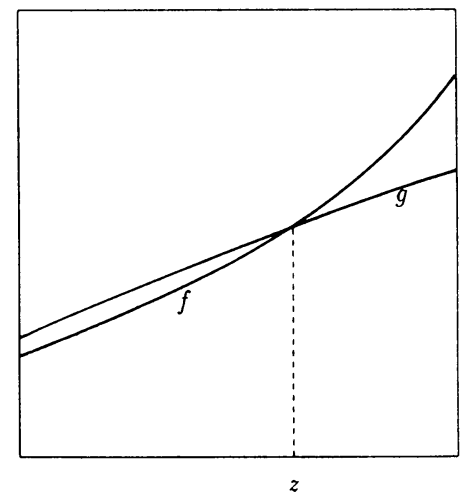

FIGURE 7. The maps $f$ and $g$

suffices to show that $A_{y}(f(y)) \geq A_{a}(f(a))$ for all $y \in[a, b)$ (recall that $A_{\mu}(x)$ denotes the $F_{\mu}$-address of $x$ ).

Lemma 2.17. Let $\underline{A}^{n}={ }^{s_{0}} d_{0} \ldots{ }^{s_{n-1}} d_{n-1}$ be an $n$-strip. Then for all $\mu \in \Delta_{\underline{A}^{n}}$ and $k=1, \ldots, n$ we have

$$
E\left(f_{k}(\mu)\right)=\sum_{i=0}^{k-1} d_{i} .
$$

Proof. Clearly $E\left(f_{k}(\mu)\right)=E\left(f_{k}(\mu)\right)-E\left(c_{\mu}\right)=\sum_{i=1}^{k}\left(E\left(f_{i}(\mu)-E\left(f_{i-1}(\mu)\right)\right)=\right.$ $\sum_{i=0}^{k-1} d_{i}(\mu)=\sum_{i=0}^{k-1} d_{i}$.

Lemma 2.18. Let $\underline{A}^{n}={ }^{C} d_{0}{ }^{L} d_{1} \ldots{ }^{L} d_{n-1}$ be an $n$-strip. Then for all $\mu \in \Delta_{\underline{A}^{n}}$ and $k=1, \ldots, n$ we have

$$
f_{k}(\mu)=\lambda^{k} c_{\mu}+p_{\underline{A}^{n}}^{k}(\lambda),
$$

where $p_{\underline{A}^{n}}^{k}(\lambda)$ is a polynomial of degree $k-1$ in $\lambda$ which depends only on $d_{0}, \ldots, d_{n-1}$.

Proof. We use induction on $k$. If $k=1$ then $f_{1}(\mu)=\lambda c_{\mu}$. Now assume that the statement holds for $k-1 \geq 1$ and we prove it for $k$. Since $\mu \in \Delta_{\underline{A}^{n}}$ and $k-$ $1 \leq n-1$ we have that $A\left(f_{k-1}(\mu)\right)={ }^{L} d_{k-1}$. Hence, by Lemma $2.17, f_{k}(\mu)=$ $F_{\mu}\left(f_{k-1}(\mu)\right)=E\left(f_{k-1}(\mu)\right)+F_{\mu}\left(f_{k-1}(\mu)-E\left(f_{k-1}(\mu)\right)\right)=\sum_{i=0}^{k-2} d_{i}+\lambda f_{k-1}(\mu)-$ $\lambda \sum_{i=0}^{k-2} d_{i}=\lambda^{k} c_{\mu}+\lambda p_{\underline{A}^{n-1}}^{k-1}(\lambda)+(1-\lambda) \sum_{i=0}^{k-2} d_{i}$ and the lemma follows.

Remark 2.19. From the preceding lemma we obtain that if

$$
\underline{A}^{n}={ }^{C} d_{0}{ }^{L} d_{1} \ldots{ }^{L} d_{n-1}
$$

then for all $\mu \in \Delta_{\underline{A}^{n}}$ and for $k=1, \ldots, n$ we have $g_{k}(\mu)=\lambda^{k} c_{\mu}^{\prime}$. Moreover in a similar way to the proof of Lemma 2.18 we also obtain that if $\underline{C}^{k}$ is a $k$-strip and $\underline{A}^{n}=\underline{C}^{k}{ }^{L} d_{k}{ }^{L} d_{k+1} \ldots{ }^{L} d_{n-1}$ then $g_{k+i}(\mu)=\lambda^{i} g_{k}(\mu)$ for $i=1, \ldots, n-k$ and for $\mu \in \Delta_{\underline{A}^{n}}$.

Remark 2.20. Since $F_{\mu}$ is old we have

$$
D\left(F_{\mu}(x)\right)=D\left(F_{\mu}(D(x))\right)
$$

for all $x \in \mathbf{R}$ and for all $\mu \in(0, \infty)$. 
Let $\underline{A}^{n}={ }^{C} d_{0} \ldots s_{n-1} d_{n-1}$ be an $n$-strip. We set $\underline{A}_{+}^{n}={ }^{R} d_{0} \ldots s_{n-1} d_{n-1}$ and $\underline{A}_{-}^{n}={ }^{L} d_{0} \ldots s_{n-1} d_{n-1}$.

Lemma 2.21. Let $\underline{A}^{n}={ }^{s_{0}} d_{0} \ldots s_{n-1} d_{n-1}$ be an n-strip such that $s_{i} \in\{L, R\}$ for $i=1, \ldots, n-1$ and assume that $\Gamma_{\underline{A}^{n}}$ is an open interval, $\left.f_{n}\right|_{\Gamma_{A^{n}}}$ is either strongly increasing and $\sigma\left(\underline{A}^{n}\right)=1$ or strongly decreasing and $\sigma\left(\underline{A}^{n}\right)=-1$; and there exists a unique $x \in \Gamma_{\underline{A}^{n}}$ such that $D\left(f_{n}(x)\right)=c_{x}$. Then the following hold.

(1) If $\sigma\left(\underline{A}^{n}\right)=1$ then for all $\mu \in \Gamma_{\underline{A}^{n}}$ such that $\mu>x, K(\mu)>\underline{A}^{n} \underline{A}_{+}^{n}\left(\underline{A}_{-}^{n}\right)^{\infty}$. Moreover, for all $l \in \mathbf{N}$ there exists $\delta_{l}$ such that $K(\mu)=\underline{A}^{n} \underline{A}_{+}^{n}\left(\underline{A}_{-}^{n}\right)^{l} \ldots$ for all $\mu \in\left(x, x+\delta_{l}\right)$.

(2) If $\sigma\left(\underline{A}^{n}\right)=-1$ then for all $\mu \in \Gamma_{\underline{A}^{n}}$ such that $\mu>x, K(\mu)>$ $\underline{A}^{n} \underline{A}_{-}^{n}\left(\underline{A}_{+}^{n}\right)^{\infty}$. Moreover, for all $l \in \mathbf{N}$ there exists $\delta_{l}$ such that $K(\mu)=$ $\underline{A}^{n} \underline{A}_{-}^{n}\left(\underline{A}_{+}^{n}\right)^{l} \cdots$ for all $\mu \in\left(x, x+\delta_{l}\right)$.

Proof. We only prove (1). Statement (2) follows in a similar way. We set $p=d_{0}+\cdots+d_{n-1}, k=\operatorname{Card}\left\{i \in\{1, \ldots, n-1\}: s_{i}=R\right\}$ and $K=\{y \in$ $\left.\Gamma_{A^{n}}: y>x\right\}$. Note that from Remark 2.5 it follows that $n=(k+1) m+r$ with $r \in \mathbf{N}$. Since $\left.f_{n}\right|_{\Gamma_{A^{n}}}$ is strongly increasing we get that $K(\mu)=\underline{A}^{n R} d \cdots$ for all $\mu \in K$. By Lemma 2.17 it follows that $f_{n}(x)=c_{x}+p$ and $p+c_{y}<f_{n}(y)<p+1$ for all $y \in K$. Set $\underline{A}_{-}=\left(\underline{A}_{-}^{n-1}\right)^{\infty}$ and $\underline{\hat{A}}_{-}=\left(d_{0}^{s_{1}} \cdots d_{n-1}^{L}\right)^{\infty}$. Note that the sequence $\underline{\hat{A}}_{-}$has no symbol $M, R$, or $C$ and $\underline{A}_{-}^{E}=\underline{A}_{-}$. Then since $s_{n}(y)=R$ for all $y \in K$, we get

$$
\left(0^{L}\right)^{\infty}<S^{i}\left(\underline{\hat{A}}_{-}\right)<\underline{\underline{I}}_{F_{y}}\left(c_{y}\right)
$$

for all $i \geq 0$. Hence $\underline{\hat{A}}_{-}$is dominated by $F_{y}$ for all $y \in K$ (see $\S 3$ of [AM] for a definition of domination). Then, by Proposition A of [AM], for a fixed $y$ there exists a periodic point $y_{1} \in\left(0, c_{y}\right)$ such that $\underline{I}_{F_{y}}\left(y_{1}\right)=\underline{A}$. Let $y_{2}$ be the conjugate of $y_{1}$ in $\left(c_{y}, 1\right)$. Clearly $F_{y}^{n}\left(y_{1}\right)-p=y_{1}$ and $\underline{I}_{F_{y}}\left(y_{2}\right)=\underline{A}_{+}^{n}\left(\underline{A}_{-}^{n}\right)^{\infty}$. Then we get that $\left.\left(F_{y}^{n}-p\right)\right|_{\left[y_{1}, y_{2}\right]}$ is piecewise linear with two pieces (see Figure 8 on next page). Moreover, by Lemma 2.3 of [AM] we get that

$$
\underline{A}_{-} \leq \underline{I}_{F_{y}}(z)<K(y)=\underline{A}^{n R} d_{0} \cdots \quad \text { for all } z \in\left[y_{1}, c_{y}\right] .
$$

Hence, for all $z \in\left[y_{1}, c_{y}\right]$, the map $\underline{I}_{F_{y}}(z)=\underline{A}_{-}^{n} \cdots$. Therefore, the slope of $\left.\left(F_{y}^{n}-p\right)\right|_{\left[y_{1}, c_{y}\right]}$ is $\lambda^{n-k} \mu^{k}$. Similarly, $\underline{I}_{F_{y}}(z)=\underline{A}_{+}^{n} \cdots$ for all $z \in\left[c_{y}, y_{2}\right]$, and the slope of $\left.\left(F_{y}^{n}-p\right)\right|_{\left[c_{y}, y_{2}\right]}$ is $\lambda^{n-k-1} \mu^{k+1}$. Since $\lambda>1, \lambda^{m-1} \mu>1$, and $1 / \lambda^{m}+1 / \lambda^{m-1} \mu<1$ for all $\mu \in I(m)$ we get

$$
\begin{aligned}
\frac{1}{\lambda^{n-k} \mu^{k}}+\frac{1}{\lambda^{n-k-1} \mu^{k+1}} & =\frac{1}{\lambda^{(m-1) k+m+r} \mu^{k}}+\frac{1}{\lambda^{(m-1) k+m+r-1} \mu^{k+1}} \\
& =\frac{1}{\left(\lambda^{m-1} \mu\right)^{k} \lambda^{r}}\left(\frac{1}{\lambda^{m}}+\frac{1}{\lambda^{m-1} \mu}\right)<1 .
\end{aligned}
$$

Hence, from Remark 2.11 we get $F_{y}^{n-1}\left(c_{y}\right)-p>y_{2}$. Therefore, $\underline{I}_{F_{y}}\left(f_{n}(y)\right)>$ $\underline{I}_{F_{y}}\left(y_{2}\right)=\underline{A}_{+}^{n}\left(\underline{A}_{-}^{n}\right)^{\infty}$. Thus, $K(y)=\underline{A}^{n} \underline{I}\left(f_{n}(y)\right)>\underline{A}^{n} \underline{A}_{+}^{n}\left(\underline{A}_{-}^{n}\right)^{\infty}$.

We note that since $D\left(f_{n}(x)\right)=c_{x}$ (that is $\left.D\left(F_{x}^{n}\left(c_{x}\right)\right)=c_{x}\right)$ we have $f_{i}(x) \notin$ $\mathbf{Z}$ for all $i \geq 0$. Moreover, since $s_{i} \in\{L, R\}$ for all $0<i<n-1$ we also 


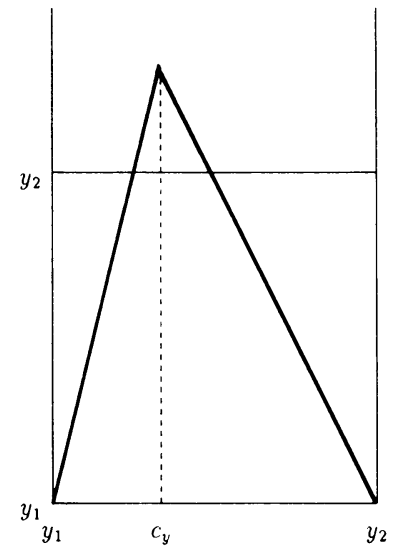

FIGURE 8. The map $F_{y}^{n}-\left.p\right|_{\left[y_{1}, y_{2}\right]}$

have $s_{i}(x) \neq C$ for all $i>0$ not a multiple of $n$. So, by the continuity of $f_{i}$ for all $i \geq 0$ it follows that for each $l \in \mathbf{N}$ there exists $\delta_{l}>0$ such that for all $y \in\left(x, x+\delta_{l}\right)$ we have $d_{i}(x)=d_{i}(y)$ for all $i \in\{0, \ldots,(n+2) l\}$ and $A_{i}(x)=A_{i}(y)$ for all $i \in\{0, \ldots,(n+2) l\}$ not a multiple of $n$. Then, since $K(y) \geq \underline{A}^{n} \underline{A}_{+}^{n}\left(\underline{A}_{-}^{n}\right)^{\infty}$ for such $y$, we get $S_{n}(y)=R$ and $s_{k n}(y)=L$ for all $k$ such that $k n<l$. This ends the proof of the lemma.

Let $\underline{B}^{n}$ be the $n$-strip formed by the first $n$ symbols of

$$
\underline{B}={ }^{C} 1\left({ }^{L} 0\right)^{m-1 R} 1\left(\left({ }^{L} 0\right)^{m-1 L} 1\right)^{\infty} .
$$

In the following lemma we show that $\Gamma_{\underline{A}^{n}}$ is an interval such that $\left.f_{n}\right|_{\Gamma_{A^{n}}}$ is strongly increasing or strongly decreasing according to the parity of $\underline{A}^{n}$. This plays a crucial role in the proof of Theorem 2.1 .

Lemma 2.22. Let $\underline{A}^{n}={ }^{s_{0}} d_{0} \ldots s_{n-1} d_{n-1}$ be an $n$-strip such that $s_{i} \in\{L, R\}$ for $i=1, \ldots, n-1$ and assume that $\Gamma_{\underline{A}^{n}} \neq \varnothing$. Then the following hold.

(1) $\Gamma_{\underline{A}^{n}}=\left(\alpha_{\underline{A}^{n}}, \beta_{\underline{A}^{n}}\right)$ with $\beta_{\underline{A}^{n}} \in \mathbf{R} \cup\{\infty\}$.

(2) Either $\bar{D}\left(f_{n}\left(\alpha_{A^{n}}\right)\right)=0$ or $c_{\alpha_{A^{n}}}$ is periodic of period less than $n$ or $\underline{A}^{n}=\underline{B}^{n}$ and in this case $\alpha_{\underline{A}^{n}}=\max \left(1 /\left(\lambda^{m}-\lambda^{m-1}-1\right), \lambda /\left(\lambda^{m}-1\right)\right)$.

(3) If $s_{i}=L$ for $i=1, \ldots, n-1$ then either $n \leq m$ and $\underline{A}^{n}=\underline{B}^{n}$ or $D\left(f_{n}\left(\alpha_{A^{n}}\right)\right)=0$ and in any case $\left.f_{n}\right|_{\Gamma_{A^{n}}}$ is strongly increasing. Otherwise, if $\sigma\left(\underline{A}^{n}\right)=1$ then $\left.f_{n}\right|_{\Gamma_{A^{n}}}$ is strongly increasing and convex and if $\sigma\left(\underline{A}^{n}\right)=-1$ then $\left.f_{n}\right|_{\Gamma_{A^{n}}}$ is strongly decreasing and concave.

Proof. To prove the lemma we use induction on $n$. First we prove the lemma for $n \leq m$ (recall that $m=1$ if $b(\mu) \geq 1$ and if $b(\mu)<1$ then $m$ is such that $\left.b(\mu) \in\left[\frac{1}{m}, \frac{1}{m-1}\right)\right)$. We divide this proof into two cases.

Case $m=1$. We have $I(m)=\left(\max \left(\frac{1}{\lambda-2}, \frac{\lambda}{\lambda-1}\right), \infty\right)$ and $\underline{B}={ }^{C} 1^{R} 1\left({ }^{L} 1\right)^{\infty}$. For $n=1$, since $\Gamma_{\underline{A}^{1}} \neq \varnothing$, we have $\underline{A}^{1}={ }^{C} d$ with $0<d<\lambda$. Clearly $\Delta_{\underline{A}^{\prime}}=\left(\frac{(d-1) \lambda}{\lambda-d}, \beta_{\underline{A}^{\prime}}\right)$ (see Figure 9) where $\beta_{\underline{A}^{\prime}}=\frac{d \lambda}{\lambda-d-1}$ if $\lambda>d+1$ or $\beta_{\underline{A}^{\prime}}=\infty$ otherwise. If $d=1$ then $\underline{A}^{1}=\underline{B}^{1}$ and we get $\alpha_{\underline{A}^{\prime}}=\max \left(\frac{1}{\lambda-2}, \frac{\lambda}{\lambda-1}\right)$. If $d>1$ 


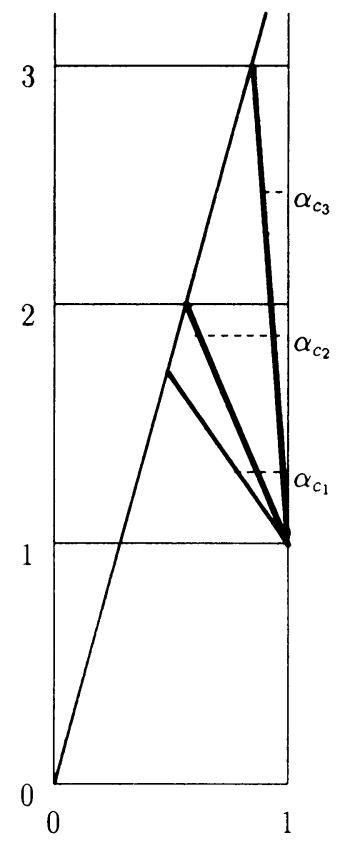

Figure 9. The values $\alpha_{C_{d}}$ for $d=1,2,3$

clearly we obtain $\alpha_{\underline{A}^{1}}=\frac{(d-1) \lambda}{\lambda-d}$ and hence $D\left(f_{1}\left(\alpha_{\underline{A}^{1}}\right)\right)=0$. Thus (1) and (2) hold.

Since $g_{1}(\mu)=\lambda c_{\mu}^{\prime}=\lambda(\lambda-1) /(\mu+\lambda)^{2}>0$ it follows that $f_{1}$ is increasing. To prove that $f_{1}$ is strongly increasing, in view Remark 2.14 , it is enough to show that $f_{1}(\mu)-c_{\mu}$ and $f_{1}(\mu)-\left(1-\frac{i}{\mu}\right)$ are increasing for $i=2, \ldots, d-1$. Clearly $g_{1}(\mu)-c_{\mu}^{\prime}>0$. To prove that $g_{1}(\mu)-i / \mu^{2}>0$ for $i=1, \ldots, d-1$ we shall show that

$$
g_{1}(\mu)=\lambda \frac{\lambda-1}{(\mu+\lambda)^{2}}>\frac{d-1}{\mu^{2}}
$$

which is equivalent to

$$
\frac{\mu \lambda}{\mu+\lambda}\left(f_{1}(\mu)-1\right)=\frac{\mu \lambda}{\mu+\lambda} \mu \frac{\lambda-1}{\mu+\lambda}>d-1 .
$$

Since $f_{1}(\mu) \geq d$ it suffices to prove that $\frac{\mu \lambda}{\mu+\lambda}>1$ which holds for $\mu>\frac{\lambda}{\lambda-1}$. Hence (3) follows for $n=1$.

Case $m>1$. We have $b(\mu)<1$ and hence $d_{i}(\mu) \in\{0,1\}$ for all $i \geq 0$. From Remark 2.5 we get that if $d_{i}(\mu)=1$ then $d_{i+1}(\mu)=\cdots d_{i+m-2}(\mu)=0$ and $s_{i+1}(\mu)=\cdots=s_{i+m-1}(\mu)=L$. First we prove the lemma for $n<m$. By Lemma 2.4 we get $\underline{A}^{n}=\underline{B}^{n}$ and $\Gamma_{\underline{A}^{n}}=I(m)$. Hence

$$
\alpha_{\underline{A}^{n}}=\max \left(1 /\left(\lambda^{m}-\lambda^{m-1}-1\right), \lambda / \lambda-1\right) .
$$

By Lemma 2.18 we have $g_{n}(\mu)=\lambda^{n} c_{\mu}^{\prime}>0$ for all $\mu \in I(m)$. Therefore, $\left.f_{n}\right|_{I(m)}$ is increasing. Lastly, from Remark 2.5 we get that $s_{n}(\mu)=L$ for all $\mu \in I(m)$. Hence $f_{n}(\mu)<c_{\mu}$ for all $\mu \in I(m)$. Therefore, $f_{n}$ is strongly increasing.

Now we prove the lemma for $n=m$. Since $\left.f_{m-1}\right|_{I(m)}$ is strongly increasing we obtain (1). By Lemma 2.4 we get that either $\underline{A}^{m}=\underline{B}^{m}$ or $\underline{A}^{m}$ - 


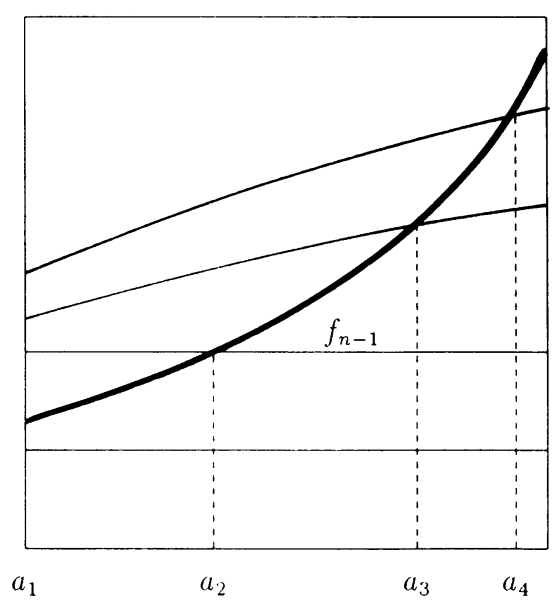

FIGURE 10. The graph of the map $\left.f_{n-1}\right|_{\Gamma_{A^{n-1}}}$ and the intervals $\Gamma_{\underline{A}^{n}}$ in different cases. Note that $\alpha_{\underline{A}^{n-1}}=a_{1}=$ $\alpha_{\underline{A}^{n-1 L_{1}}}, \alpha_{\underline{A}^{n-1 L 2}}=a_{2}$ and, by Remark 2.20, $D\left(f_{n}\left(a_{2}\right)\right)$ $=D\left(F_{a_{2}}\left(D\left(f_{n-1}\left(a_{2}\right)\right)\right)=D\left(F_{a_{2}}\left(\frac{1}{\lambda}\right)\right)=0 ; \alpha_{\underline{A}^{n-1} R_{2}}=a_{3}\right.$ and $D\left(f_{n-1}\left(a_{3}\right)\right)=c_{a_{3}}$, and finally $\alpha_{\underline{A}^{n-1 R}}=a_{4}$ and $D\left(f_{n}\left(a_{4}\right)\right)=D\left(F_{a_{4}}\left(f_{n-1}\left(a_{4}\right)\right)\right)=D\left(F_{a_{4}}\left(1-\frac{1}{a_{4}}\right)\right)=0$

${ }^{C} 1\left({ }^{L} 0\right)^{m-2 L} 1$. Clearly, in the first case $\alpha_{\underline{A}^{m}}=\max \left(1 /\left(\lambda^{m}-\lambda^{m-1}-1\right), \lambda / \lambda-1\right)$. If $\underline{A}^{m}={ }^{C} 1\left({ }^{L} 0\right)^{m-2 L} 1$ we get that $f_{m}\left(\alpha_{\underline{A}^{m}}\right)=2$. Hence (2) holds. Now we prove (3). From Lemma 2.18 we have $g_{m}(\mu)=\lambda^{m} c_{\mu}^{\prime}>0$ for all $\mu \in \Gamma_{\underline{A}^{m}}$. Therefore $\left.f_{m}\right|_{\Gamma^{m}}$ is increasing. If $\underline{A}^{m}={ }^{C} 1\left({ }^{L} 0\right)^{m-2 L} 1$, from Remark 2.5 $s_{m}(\mu)=L$ for all $\mu \in \Gamma_{\underline{A}^{m}}$. So $\left.f_{m}\right|_{\Gamma_{\underline{A}}^{m}}$ is strongly increasing. Assume that $\underline{A}^{m}=\underline{B}^{m}$. Then by Lemma 2.9 it follows that $s_{m}(\mu)=R$ for all $\mu \in \Gamma_{\underline{A}^{m}}$. Thus $\left.f_{m}\right|_{\Gamma_{\underline{A}} m}$ is strongly increasing. This ends the proof of the lemma for $n \leq m$.

Now assume that the lemma holds for $n-1 \geq m$ and we prove it for $n$. Let $\underline{A}^{n}=A_{0} A_{1} \cdots A_{n-1}$ be such that $\Gamma_{\underline{A}^{n}} \neq \varnothing$ and let $\underline{A}^{n-1}=A_{0} \cdots A_{n-2}$. First we note that since $\Gamma_{\underline{A}^{n}} \subset \Gamma_{\underline{A}^{n-1}}$ and $\left.D \circ f_{n-1}\right|_{\Gamma_{A^{n-1}}}$ is strongly increasing or strongly decreasing according to $\sigma\left(\underline{A}^{n-1}\right)$ we obtain that the set of $\mu \in \Gamma_{\underline{A}^{n-1}}$ such that $A\left(F_{\mu}\left(D\left(f_{n-1}(\mu)\right)\right)\right)={ }^{s_{n-1}} d_{n-1}$ is connected (see Figure 10). Hence (1) follows.

Now we prove (2). If $D\left(f_{n}\left(\alpha_{\underline{A}^{n}}\right)\right) \neq 0$ then either $D\left(f_{n-1}\left(\alpha_{\underline{A}^{n}}\right)\right)=c_{\alpha_{\underline{A}^{n}}}$ or $\alpha_{\underline{A}^{n}}=\alpha_{\underline{A}^{n-1}}$ (see Figure 10). In the first case $c_{\alpha_{A^{n}}}$ is periodic of period smaller than or equal to $n-1$. If $\alpha_{\underline{A}^{n}}=\alpha_{\underline{A}^{n-1}}$ and $\bar{D}\left(f_{n-1}\left(\alpha_{\underline{A}^{n-1}}\right)\right)=0$, then from Remark 2.20 we get $D\left(f_{n}\left(\alpha_{\underline{A}^{n}}\right)\right)=D\left(F_{\alpha_{\underline{A}}^{n}}\left(D\left(f_{n-1}\left(\alpha_{\underline{A}^{n-1}}\right)\right)\right)\right)=D\left(F_{\alpha_{\underline{\underline{A}}}^{n}}(0)\right)=0$. Also if $\alpha_{\underline{A}^{n}}=\alpha_{\underline{A}^{n-1}}$ and $c_{\alpha_{\underline{A}}^{n-1}}$ is periodic of period less than $n-1$ then $c_{\alpha_{\alpha^{n}}}$ is periodic of period less than $n$. By the induction hypotheses the only remaining case is $\alpha_{\underline{A}^{n}}=\alpha_{\underline{A}^{n-1}}, \underline{A}^{n-1}=\underline{B}^{n-1}$, and

$$
\alpha_{\underline{A}^{n-1}}=\max \left(1 /\left(\lambda^{m}-\lambda^{m-1}-1\right), \lambda /\left(\lambda^{m}-1\right)\right) .
$$


If $\max \left(1 /\left(\lambda^{m}-\lambda^{m-1}-1\right), 1 /\left(\lambda^{m}-1\right)\right)=\lambda /\left(\lambda^{m}-1\right)$, from Lemma 2.9 we get $K\left(\alpha_{\underline{A}^{n-1}}\right)=K\left(\lambda /\left(\lambda^{m}-1\right)\right)=\underline{B}$. Hence $s_{i}\left(\alpha_{\underline{A}^{n-1}}\right) \in\{L, R\}$ and $f_{i}\left(\alpha_{\underline{A}^{n-1}}\right) \notin$ $\mathbf{Z}$ for all $i \geq 1$. Then, from the continuity of $f_{n-1}$ we get $d_{n-1}(\mu)=$ $d_{n-1}\left(\alpha_{\underline{A}^{n-1}}\right)=d_{n-1}\left(\lambda /\left(\lambda^{m}-1\right)\right)$ and $s_{n-1}(\mu)=s_{n-1}\left(\alpha_{\underline{A}^{n-1}}\right)=s_{n-1}\left(\lambda /\left(\lambda^{m}-1\right)\right)$ for $\mu>\alpha_{\underline{A}^{n-1}}$ sufficiently close to $\alpha_{\underline{A}^{n-1}}$. Then we obtain $\underline{A}^{n}=\underline{B}^{n}$. If

$$
\max \left(1 /\left(\lambda^{m}-\lambda^{m-1}-1\right), \lambda /\left(\lambda^{m}-1\right)\right)=1 /\left(\lambda^{m}-\lambda^{m-1}-1\right)
$$

then $\lambda \leq \pi_{m}$ and, from Lemma 2.4, we obtain

$$
K\left(\alpha_{\underline{A}^{n-1}}\right)=K\left(1 /\left(\lambda^{m}-\lambda^{m-1}-1\right)\right)=\left({ }^{C} 1\left({ }^{L} 0\right)^{m-1}\right)^{\infty} .
$$

From the continuity of $f_{i}$ for $i \geq 0$ and since $f_{i}\left(\alpha_{\underline{A}^{n-1}}\right) \notin \mathbf{Z}$ for $i \geq 0$ we get $d_{i}(\mu)=d_{i}\left(\alpha_{A^{n-1}}\right)$ for $\mu$ sufficiently close to $\alpha_{\underline{A}^{n-1}}$. If $i$ is not a multiple of $m$ then $s_{n-1}\left(\alpha_{\underline{A}^{n-1}}\right)=L$. Again by the continuity of $f_{i}, s_{i}(\mu)=L$ for $\mu$ sufficiently close to $\alpha_{\underline{A}^{n-1}}$ and $i$ less than $n$, not a multiple of $m$. If $i=k \mathrm{~m}$ with $k \in \mathbf{N}$, by Lemma 2.9, we get $K(\mu)>\underline{B}$ for all $\mu \in \Gamma_{\underline{A}^{n}}$. So,

$$
s_{i}(\mu)= \begin{cases}R & \text { if } k=1, \\ L & \text { if } k>1,\end{cases}
$$

for $\mu$ sufficiently close to $\alpha_{\underline{A}^{n-1}}$ and for $i$ less than $n$ and multiple of $m$. Then $\underline{A}^{n}=\underline{B}^{n}$ and (2) holds.

Now we prove (3). We divide this proof into five cases.

Case (A). $s_{i}=L$ for $i=1, \ldots, n-2$. First we study simultaneously the cases $m=1$ and $d_{0}>1$, and $m>1$ and $d_{m-1}=1$. By the induction hypotheses we have that $D\left(f_{n-1}\left(\alpha_{\underline{A}^{n-1}}\right)\right)=0$. If $s_{n-1}=L$ then $\alpha_{\underline{A}^{n}}$ is such that $\alpha_{\underline{A}^{n}} \in \Gamma_{\underline{A}^{n-1}}, D\left(f_{n-1}\left(\alpha_{\underline{A}^{n}}\right)\right)<c_{\alpha_{\underline{A}^{n}}}$, and $F_{\alpha_{A^{n}}}\left(D\left(f_{n-1}\left(\alpha_{\underline{A}^{n}}\right)\right)\right)^{\bar{m}}=d_{n-1}$. Hence, $D\left(f_{n-1}\left(\alpha_{\underline{A}^{n}}\right)\right)=d_{n-1} / \bar{\lambda}$ (see Figure 11). By Remark 2.20, $D\left(f_{n}\left(\alpha_{\underline{A}^{n}}\right)\right)=$ $D\left(F_{\alpha_{\underline{A}^{n}}}\left(D\left(f_{n-1}\left(\alpha_{\underline{A}^{n}}\right)\right)\right)\right)=0$. From Remark 2.19 we also obtain

$$
\left.g_{n}\right|_{\Gamma_{\underline{A}^{n}}}=\lambda^{n} c_{\mu}^{\prime}>\lambda c_{\mu}^{\prime}=\left.g_{1}\right|_{\Gamma_{\underline{A}^{n}}} .
$$

Therefore, if $c_{k}(\mu)$ denotes the $k$ th turning point of $F_{\mu}$ we get

$$
g_{n}(\mu)-c_{k}(\mu)>g_{1}(\mu)-c_{k}(\mu)>0
$$

for all $\mu \in \Gamma_{\underline{A}^{n}}$.

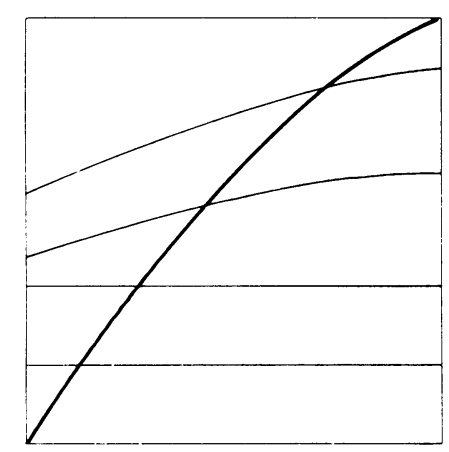

FIgURE 11. The map $\left.f_{n-1}\right|_{\Gamma_{A^{n-1}}}$ in Case (A) 
If $s_{n-1}=R$ then there exists $\mu \in \Gamma_{A^{n-1}}$ such that $D\left(f_{n-1}(\mu)\right)>c_{\mu}$. Since $D\left(f_{n-1}\left(\alpha_{\underline{A}^{n-1}}\right)\right)=0$ there exists $x \in \bar{\Gamma}_{\underline{A}^{n-1}}$ such that $D\left(f_{n-1}(x)\right)=c_{x}$ and $K(x)=\left(\underline{A}^{n-1}\right)^{\infty}$. Moreover, from Remark 2.19, $g_{n-1}(\mu)=\lambda^{n-1} c_{\mu}^{\prime}>c_{\mu}^{\prime}$ for all $\mu \in \Gamma_{\underline{A}^{n-1}}$. Therefore, for all $\mu \in\left(x, \beta_{\underline{A}^{n-1}}\right)$ we get $K(\mu)=\underline{A}^{n-1} R d_{n-1}$. Now we show that $\left.f_{n}\right|_{\left(x, \beta_{A^{n-1}}\right)}$ is concave. From Remark 2.19 we have $g_{n-1}(\mu)=$ $\lambda^{n-1} c_{\mu}^{\prime}=\lambda^{n-1}(\lambda-1) /(\mu+\lambda)^{2}$. By Remark 2.20 we also have

$$
D\left(f_{n}(\mu)\right)=D\left(F_{\mu}\left(D\left(f_{n-1}(\mu)\right)\right)=D\left(\mu\left(1-D\left(f_{n-1}(\mu)\right)\right)+E\left(f_{n-1}(\mu)\right)\right) .\right.
$$

Hence,

$$
g_{n}(\mu)=1-D\left(f_{n-1}(\mu)\right)-\mu g_{n-1}=1-D\left(f_{n-1}(\mu)\right)-\mu \lambda^{n-1} \frac{\lambda-1}{(\mu+\lambda)^{2}} .
$$

Thus,

$$
g_{n}^{\prime}(\mu)=-2 g_{n-1}+2 \mu \lambda^{n-1} \frac{\lambda-1}{(\mu+\lambda)^{3}}=-2 \lambda^{n} \frac{\lambda-1}{(\mu+\lambda)^{3}}<0 .
$$

Hence $\left.f_{n}\right|_{\left(x, \beta_{A^{n-1}}\right)}$ is concave as it was claimed. Therefore to see that $\left.f_{n}\right|_{\left(x, \beta_{\mathbb{A}^{n-1}}\right)}$ is decreasing it suffices to show that $g_{n}(x) \leq 0$.

Now suppose that $g_{n}(x)>0$. By Lemma 2.21, for all $k>1$ there exists $\delta_{k}>0$ such that $K(y)=\underline{A}^{n-1} \underline{A}_{+}^{n-1}\left(\underline{A}_{-}^{n-1}\right)^{k} \cdots$ for all $y \in\left(x, x+\delta_{k}\right)$. Then, by using Remark 2.19 we have

$$
g_{(n-1)(k+2)}(x)=\lambda^{(n-1)(k+1)-1} g_{n}(x) .
$$

Therefore, for $k$ sufficiently large we obtain

$$
g_{(n-1)(k+2)}(x)>(\lambda-1) /\left(x+\lambda^{2}\right)=c_{x}^{\prime} .
$$

Since $c_{x}$ is periodic of period a divisor of $n-1$ we get $D\left(f_{(n-1)(k+2)}(x)\right)$ $=c_{x}$ for such $k$. Since the functions $c_{\mu}$ and $D\left(f_{(n-1)(k+2)}(\mu)\right)$ coincide at $\mu=$ $x$ and $g_{(n-1)(k+2)}(x)>(\lambda-1) /(x+\lambda)^{2}=c_{x}^{\prime}>0$ we have $D\left(f_{(n-1)(k+2)}(y)\right)>c_{y}$ for $y>x$ sufficiently close to $x$. Therefore, $K(y)=\underline{A}^{n-1} \underline{A}_{+}^{n-1}\left(\underline{A}_{-}^{n-1}\right)^{k R} d \ldots$ which is a contradiction with the above claim. Hence, $g_{n}(x) \leq 0$ and $\left.f_{n}\right|_{\left(x, \beta_{A^{n-1}}\right)}$ is decreasing. So it is strongly decreasing and $(3)$ holds.

It remains to consider the cases $m=1$ and $d_{0}=1$, and $m>1$ and $d_{m-1}=$ 0 (recall again that when $m>1, d_{i}(\mu) \in\{0,1\}$ for all $i \geq 0$ ). By Lemma 2.4 it follows that $n=m+1$ and $\underline{A}^{m+1}={ }^{C} 1\left({ }^{L} 0\right)^{m-1 R} 1$ because $\Gamma_{\underline{A}^{n}} \neq \varnothing$. Clearly $\alpha_{\underline{A}^{m+1}}=\alpha_{\underline{A}^{m}}=\max \left(1 /\left(\lambda^{m}-\lambda^{m-1}-1\right), \lambda /\left(\lambda^{m}-1\right)\right)$. By the same arguments as above $\left.\bar{f}_{m+1}\right|_{\Gamma_{A} m+1}$ is concave. Then, to prove that $\left.f_{m+1}\right|_{\underline{A}^{m+1}}$ is decreasing it suffices to show that $g_{m+1}\left(\alpha_{\underline{A}^{m+1}}\right) \leq 0$. By Remark 2.5 it follows $s_{m+1}\left(\alpha_{\underline{A}^{m+1}}\right)=$ $\cdots=s_{2 m-1}\left(\alpha_{\underline{A}^{m+1}}\right)=L$. Then, from Remark 2.19 we obtain that $g_{m+1}\left(\alpha_{\underline{A}^{m+1}}\right)$ and $g_{2 m}\left(\alpha_{\underline{A}^{m+1}}\right)$ have the same sign. Now we compute the sign of $g_{2 m}\left(\alpha_{\underline{A}^{m+1}}\right)$. Assume that $\max \left(1 /\left(\lambda^{m}-\lambda^{m-1}-1\right), \lambda /\left(\lambda^{m}-1\right)\right)=1 /\left(\lambda^{m}-\lambda^{m-1}-1\right)$. We have

$$
D\left(f_{2 m}\left(\alpha_{\underline{A}^{m+1}}\right)\right)=c_{\alpha_{\underline{A}}^{m+1}}=\frac{1 /\left(\lambda^{m}-\lambda^{m-1}-1\right)+1}{1 /\left(\lambda^{m}-\lambda^{m-1}-1\right)+\lambda}=\frac{\lambda^{m-1}}{\lambda^{m}-1} .
$$

By Lemma 2.9, $K(\mu)>{ }^{C} 1\left({ }^{L} 0\right)^{m-1 R} 1\left({ }^{L} 0\right)^{m-1}\left({ }^{L} 1\left({ }^{L} 0\right)^{m-1}\right)^{\infty}$ for all $\mu \in$ $\Gamma_{\underline{A}^{m+1}}$. Hence, $\underline{I}_{F_{\mu}}\left(f_{2 m}(\mu)\right)<\left({ }^{L} 1\left({ }^{L} 0\right)^{m-1}\right)^{\infty}$ for all $\mu \in \Gamma_{\underline{A}^{m+1}}$. Since

$$
\underline{I}_{F_{\mu}}\left(\lambda^{m-1} /\left(\lambda^{m}-1\right)\right)=\left({ }^{L} 1\left({ }^{L} 0\right)^{m-1}\right)^{\infty},
$$


from Lemma 2.3 of [AM] we obtain $f_{2 m}(\mu)<\lambda^{m-1} /\left(\lambda^{m}-1\right)$ for all $\mu \in \Gamma_{\underline{A}^{m+1}}$ and hence, $g_{2 m}\left(\alpha_{\underline{A}^{m+1}}\right) \leq 0$. Now assume that

$$
\max \left(1 /\left(\lambda^{m}-\lambda^{m-1}-1, \lambda /\left(\lambda^{m}-1\right)\right)=\lambda /\left(\lambda^{m}-1\right) .\right.
$$

Then,

$$
D\left(f_{2 m}\left(\alpha_{\underline{A}^{m+1}}\right)\right)=\frac{1}{\lambda^{m}-1} .
$$

As above, by Lemma 2.9 we get $K(\mu)>{ }^{C} 1\left({ }^{L} 0\right)^{m-1 R} 1\left({ }^{L} 0\right)^{m-1}\left({ }^{L} 1\left({ }^{L} 0\right)^{m-1}\right)^{\infty}$ for all $\mu \in \Gamma_{\underline{A}^{m+1}}$ and, hence, $\underline{I}_{F_{\mu}}\left(f_{2 m}(\mu)\right)<\left({ }^{L} 1\left({ }^{L} 0\right)^{m-1}\right)^{\infty}$ for all $\mu \in \Gamma_{\underline{A}^{m+1}}$. Since $\underline{I}_{F_{\mu}}\left(1 /\left(\lambda^{m}-1\right)\right)=\left({ }^{L} 1\left({ }^{L} 0\right)^{m-1}\right)^{\infty}$, from Lemma 2.3 of [AM] we obtain $f_{2 m}(\mu)<1 /\left(\lambda^{m}-1\right)$ for all $\mu \in \Gamma_{\underline{A}^{m+1}}$. Then $g_{2 m}\left(\alpha_{\underline{A}^{m+1}}\right) \leq 0$. Thus, $\left.f_{m+1}\right|_{\Gamma_{\underline{A}}^{m+1}}$ is decreasing and hence it is strongly decreasing.

Case (B). $\sigma\left(\underline{A}^{n-1}\right)=1, s_{j}=R$ for some $j \in\{1, \ldots, n-2\}$, and $s_{n-1}=$ $L$. Since $\sigma\left(\underline{A}^{n-1}\right)=1$ by the induction hypotheses we know that $f_{n-1} \mid \Gamma_{A^{n-1}}$ is strongly increasing and convex. Thus $A\left(f_{n-1}\left(\alpha_{A^{n-1}}\right)\right) \leq s_{n-1} d_{n-1}$ because $\Gamma_{A^{n}} \subset$ $\Gamma_{\underline{A}^{n-1}}$. Since $s_{n-1}=L$ we get $s_{n-1}\left(\alpha_{\underline{A}^{n-1}}\right) \in\{\bar{M}, L\}$ and $d_{n-1}\left(\alpha_{\underline{A}^{n-1}}\right) \leq \bar{d}_{n-1}$. Furthermore, from Remark 2.20 it follows that $D\left(f_{n}(x)\right)=D\left(F_{x}\left(D\left(f_{n-1}(x)\right)\right)\right)$ $=D\left(\lambda D\left(f_{n-1}(x)\right)\right.$ for all $x \in \Gamma_{\underline{A}^{n}}$. Hence, $\left.g_{n}\right|_{\Gamma_{A^{n}}}=\left.\lambda g_{n-1}\right|_{\Gamma_{A^{n}}}$ and $\left.g_{n}^{\prime}\right|_{\Gamma_{\underline{A}^{n}}}=$ $\lambda g_{n-1}^{\prime} \mid \Gamma_{A^{n}}$. Thus $\left.f_{n}\right|_{\Gamma_{A^{n}}}$ is increasing and convex. Hence only it remains to prove that $\left.f_{n}\right|_{\Gamma_{A^{n}}}$ is strongly increasing.

If $d_{n-1}\left(\alpha_{\underline{A}^{n-1}}\right)<d_{n-1}$ we get $D\left(f_{n}\left(\alpha_{\underline{A}^{n}}\right)\right)=0$ (see Figure 12). Then, since the functions $(\mu+1) /(\mu+\lambda)$ and $1-k / \mu$ for $k \in \mathbf{N}$ are increasing and concave, by Lemma 2.15 , we obtain that $\left.D \circ f_{n}\right|_{\Gamma_{A^{n}}}$ is strongly increasing.

Now we assume that $d_{n-1}\left(\alpha_{\underline{A}^{n-1}}\right)=\bar{d}_{n-1}$. Then $\alpha_{\underline{A}^{n}}=\alpha_{\underline{A}^{n-1}}$. If

$$
D\left(f_{n-1}\left(\alpha_{\underline{A}^{n-1}}\right)\right)=0
$$

we obtain that $\left.D \circ f_{n}\right|_{\Gamma_{A^{n}}}$ is strongly increasing by the same arguments as above. Hence we assume that $D\left(f_{n-1}\left(\alpha_{\underline{A}^{n-1}}\right)\right) \neq 0$. If $s_{n}\left(\alpha_{\underline{A}^{n}}\right)=L$ we obtain (3) from Lemma 2.15. Therefore, we also assume that $s_{n}\left(\alpha_{\underline{A}^{n}}\right) \in\{C, R\}$. Since $\sigma\left(\underline{A}^{n-1}\right)=1$ and there exists $j \in\{1, \ldots, n-2\}$ such that $s_{j}=R$ we get

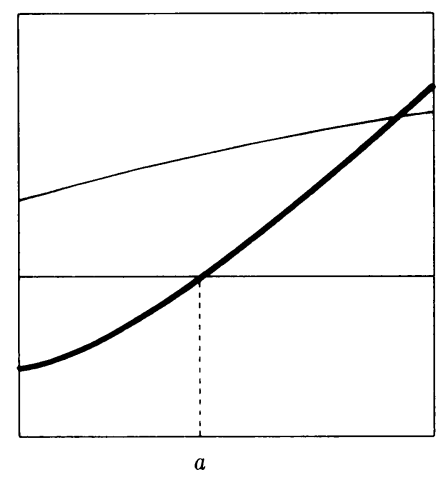

FIGURE 12. The map $\left.f_{n-1}\right|_{\Lambda_{A^{n-1}}}$ in Case (B) when $m>$ 1 . Note that $f_{n-1}(a)=i / \lambda$ and hence $D\left(f_{n}(a)\right)=0$ 
$\underline{A}^{n-1} \neq \underline{B}^{n-1}$. By the induction hypotheses we have that $c_{\alpha_{\underline{A}^{n}}}=c_{\alpha_{A^{n-1}}}$ is periodic of period $k<n-1$. Set $\underline{A}^{k}=A_{0} \cdots A_{k-1}$ and consider two subcases:

Subcase (B1) $\sigma\left(\underline{A}^{k}\right)=1$. By the induction hypotheses we have that $\left.f_{k}\right|_{\Gamma_{A^{k}}}$ is strongly increasing. Since $\sigma\left(\underline{A}^{k}\right)=1$, from Lemma 2.21 we get $K(y)>$ $\underline{A}^{k} \underline{A}_{+}^{k}\left(\underline{A}_{-}^{k}\right)^{\infty}$ for all $y>\alpha_{\underline{A}^{n}}$. Therefore, either $\underline{A}^{n}=\underline{A}^{k} \underline{A}_{+}^{k}\left(\underline{A}_{-}^{k}\right)^{j L} d_{0} A_{1} \cdots A_{i}$ for some $j \geq 0$ and $0<i<k-1$ or $\underline{A}^{n}=\underline{A}^{k R} d_{0} A_{1} \cdots A_{i}$ for some $0<i<k-$ 1 or $\underline{A}^{n}=\underline{A}^{k} \underline{A}_{+}^{k}\left(\underline{A}_{-}^{k}\right)^{j}$ for some $j \geq 0$. Since $\sigma\left(\underline{A}^{n}\right)=1$ and $\sigma\left(\underline{A}^{k} \underline{A}_{+}^{k}\left(\underline{A}_{-}^{k}\right)^{j}\right)=$ -1 the last possibility cannot occur and in both of the remaining cases we have $\sigma\left(A_{1} \cdots A_{i}\right)=-1$. In view of Remark 2.16 , to show that $\left.f_{n}\right|_{\Gamma_{A^{n}}}$ is strongly increasing it suffices to prove that $A\left(f_{n}(y)\right) \geq A\left(f_{n}\left(\alpha_{\underline{A}^{n}}\right)\right)=A_{i+1}$ for all $y \in$ $\Gamma_{\underline{A}^{n}}$. In the first case we have

$$
K(y)=A_{0} A_{1} \cdots A_{i} A_{i+1} \cdots A_{n-1} \underline{A}_{+}^{k}\left(\underline{A}_{-}^{k}\right)^{j L} d_{0} A_{1} \cdots A_{i} A_{n}(y) \cdots
$$

for all $y \in \Gamma_{\underline{A}^{n}}$. Hence,

$$
\underline{I}_{F_{y}}\left(F_{y}^{(j+2) k}\left(c_{y}\right)\right)=S^{(j+2) k}(K(y))={ }^{L} d_{0} A_{1} \cdots A_{i} A_{n}(y) \cdots \leq \underline{I}_{F_{y}}\left(c_{y}^{-}\right) .
$$

Since $A_{0}={ }^{C} d_{0}$ and $\sigma\left(A_{1} \cdots A_{i}\right)=-1$ we obtain $A_{n}(y)=A\left(f_{n}(y)\right) \geq$ $A_{i+1}=A\left(f_{n}\left(\alpha_{\underline{A}^{n}}\right)\right)$. In the second case,

$$
K(y)=A_{0} A_{1} \cdots A_{i} A_{i+1} \cdots A_{n-1}{ }^{R} d_{0} A_{1} \cdots A_{i} A_{n}(y)
$$

for all $y \in \Gamma_{\underline{A}^{n}}$. Therefore

$$
\underline{I}_{F_{y}}\left(F_{y}^{k}\left(c_{y}\right)\right)=S^{k}(K(y))={ }^{R} d_{0} A_{1} \cdots A_{i} A_{n}(y) \cdots \geq \underline{I}_{F_{y}}\left(c_{y}^{+}\right) .
$$

Since $A_{0}={ }^{C} d_{0}$ and $\sigma\left(A_{1} \cdots A_{i}\right)=-1$ we get $A_{n}(y)=A\left(f_{n}(y)\right) \geq A_{i+1}=$ $A\left(f_{n}\left(\alpha_{\underline{A}^{n}}\right)\right)$.

Subcase (B2). $\sigma\left(\underline{A}^{k}\right)=-1$. By the induction hypotheses, $\left.f_{k}\right|_{\Gamma_{A^{k}}}$ is strongly decreasing. We shall prove that $A_{n}(y) \geq A_{n}\left(\alpha_{\underline{A}^{n}}\right)$ for all $y \in \underline{A}^{n}$. From Lemma 2.21 we obtain

$$
K(y)>\underline{A}^{k} \underline{A}_{-}^{k}\left(\underline{A}_{+}^{k}\right)^{\infty} \quad \text { for all } y>\alpha_{\underline{A}^{n}} .
$$

Thus, either $\underline{A}^{n}=\underline{A}^{k} \underline{A}_{-}^{k}\left(\underline{A}_{+}^{k}\right)^{j R} d_{0} A_{1} \cdots A_{i}$ for some $j \geq 0$ and $i>0$ or $\underline{A}^{n}=\underline{A}^{k L} d_{0} A_{1} \cdots A_{i}$ for some $i>0$ or $\underline{A}^{n}=\underline{A}^{k} \underline{A}_{-}^{k}\left(\underline{A}_{+}^{k}\right)^{j}$ for some $j \geq 0$. In the first and in the second case we have $\sigma\left(A_{1} \cdots A_{i}\right)=-1$ and we obtain the desired result as in Subcase (B1). In the last case we note that $s_{n}\left(\alpha_{\underline{A}^{n}}\right)=C$, and by $(\diamond)$ we have $s_{n}(y)=R$ for all $y \in \Gamma_{\underline{A}^{n}}$. Therefore, $f_{n}$ is strongly increasing in $\Gamma_{\underline{A}^{n}}$.

Case (C). $\sigma\left(\underline{A}^{n-1}\right)=-1$ and $s_{n-1}=L$. Since $g_{n}=\lambda g_{n-1}$ and $g_{n}^{\prime}=\lambda g_{n-1}^{\prime}$, $\left.f_{n}\right|_{\Gamma_{A^{n}}}$ is decreasing and concave because $\left.f_{n-1}\right|_{\Gamma_{A^{n-1}}}$ is decreasing and concave by the induction hypotheses.

Case (D). $\sigma\left(\underline{A}^{n-1}\right)=-1$ and $s_{n-1}=R$. Since $g_{n}(\mu)=\left(1-D\left(f_{n-1}(\mu)\right)\right)-$ $\mu g_{n-1}(\mu)$ and $g_{n}^{\prime}(\mu)=-2 g_{n-1}(\mu)-\mu g_{n-1}^{\prime}(\mu),\left.f_{n}\right|_{\Gamma_{A^{n}}}$ is increasing and convex. To see that $f_{n}$ is strongly increasing we use similar arguments to those of Case (B). 
Case (E). $\sigma\left(\underline{A}^{n-1}\right)=1, s_{j}=R$ for some $j \in\{1, \ldots, n-2\}$ and $s_{n-1}=R$. By the induction hypotheses we know that $\left.f_{n-1}\right|_{\Gamma_{A} n-1}$ is strongly increasing. Hence, there exists an interval $K$ such that $\Gamma_{\underline{A}^{n}} \subset K^{\underline{A}} \subset \Gamma_{\underline{A}^{n-1}}$ and $s_{n-1}(\mu)=R$ for all $\mu \in K$ (recall that $\left.\Gamma_{\underline{A}^{n}} \neq \varnothing\right)$. Clearly $g_{n}(\mu)=1-D\left(f_{n-1}(\mu)\right)-\mu g_{n-1}(\mu)$ and $g_{n}^{\prime}(\mu)=-2 g_{n-1}(\mu)-g_{n-1}^{\prime}(\mu)$ for all $\mu \in K$. Since, by the induction hypotheses, $\left.g_{n-1}\right|_{\Gamma^{n-1}}>0$ and $\left.g_{n-1}^{\prime}\right|_{\Gamma_{A^{n-1}}}>0$ we obtain that $\left.f_{n}\right|_{K}$ is concave. To see that $\left.f_{n}\right|_{\Gamma_{A^{n}}}$ is decreasing (i.e., strongly decreasing) we consider again two subcases.

Subcase (E1). $s_{n-1}\left(\alpha_{\underline{A}^{n-1}}\right) \in\{M, L\}$. Since $s_{n-1}=R$, there exists $x \in \Gamma_{\underline{A}^{n-1}}$ such that $f_{n-1}(x)=c_{x}$. Clearly $K=\left(x, \beta_{\underline{A}^{n-1}}\right)$. Then, since $\left.f_{n}\right|_{K}$ is concave, to prove that $\left.f_{n}\right|_{\Gamma_{A^{n}}}$ is decreasing it suffices to show that $g_{n}(x) \leq 0$.

From Lemma 2.21 we have that $K(y)>\underline{A}^{n-1} \underline{A}_{+}^{n-1}\left(\underline{A}_{-}^{n-1}\right)^{\infty}$ for all $y \in K$. Moreover, for each $l \in \mathbf{N}$ there exists $\delta_{l}$ such that $K(y)=\underline{A}^{n-1} \underline{A}_{+}^{n-1}\left(\underline{A}_{-}^{n-1}\right)^{l} \ldots$ for all $y \in\left(x, x+\delta_{l}\right)$.

Set $Q_{i}\left(c_{\mu}\right)=f_{i}(\mu)=F_{\mu}^{i}\left(c_{\mu}\right)$ for $i \geq 1$. Note that $g_{i}(\mu)=Q_{i}^{\prime}\left(c_{\mu}\right) c_{\mu}^{\prime}$, and since $c_{\mu}^{\prime}>0, Q_{i}^{\prime}\left(c_{\mu}\right)$ has the same sign as $g_{i}(\mu)$ for all $i>0$. Moreover, we also note that if $K(y)={ }^{C} d_{0} A_{1} \cdots A_{j} \cdots$ and $\underline{I}_{F_{y}}(z)={ }^{L} d_{0} \cdots A_{j} \cdots$, then $F_{y}^{i}(z)=Q_{i}(z)$ for $i=1, \ldots, j+1$. These properties will be used later.

We recall that for $y \in K$ we have $s_{n-1}(y)=R$. Hence $D\left(f_{n-1}(y)\right)>$ $c_{y}$. Now, for such $y$, we denote by $\tilde{f}_{n-1}(y)$ the conjugate of $D\left(f_{n-1}(y)\right)$ in $\left(0, c_{y}\right]$. By the definition of the conjugate of a point we have $\lambda \tilde{f}_{n-1}(y)=$ $y\left(1-D\left(f_{n-1}(y)\right)\right)+1$. Hence,

$$
\tilde{f}_{n-1}(y)=\frac{1+y\left(1-D\left(f_{n}(y)\right)\right.}{\lambda} .
$$

On the other hand, if $y>x$ is sufficiently close to $x$ we have $K(y)=$ $\underline{A}^{n-1} \underline{A}_{+}^{n-1} \cdots$. Hence, by Remark 2.20,

$$
D\left(f_{n-1+k}(y)\right)=D\left(F_{y}^{k}\left(D\left(f_{n-1}(y)\right)\right)\right)=D\left(F_{y}^{k}\left(\tilde{f}_{n-1}(y)\right)\right)=D\left(Q_{k}\left(\tilde{f}_{n-1}(y)\right)\right)
$$

for $k=1, \ldots, n-1$. Thus,

$$
\begin{aligned}
g_{n-1+k}(y) & =Q_{k}^{\prime}\left(\tilde{f}_{n-1}(y)\right)\left(\tilde{f}_{n-1}(y)\right)^{\prime} \\
& =Q_{k}^{\prime}\left(\tilde{f}_{n-1}(y)\right) \frac{1-D\left(f_{n-1}(y)\right)-y g_{n-1}(y)}{\lambda} \\
& =\frac{1}{\lambda} Q_{k}^{\prime}\left(\tilde{f}_{n-1}(y)\right) g_{n}(y) .
\end{aligned}
$$

In particular, for $y>x$ sufficiently close to $x$ we obtain $g_{2(n-1)}(y)=$ $\frac{1}{\lambda} Q_{n-1}^{\prime}\left(\tilde{f}_{n-1}(y)\right) g_{n}(y)$. We also note that for $y \in\left[x, x+\delta_{1}\right)$ and $k=1, \ldots$, $n-1$ we have

and

$$
D\left(f_{2(n-1)+k}(y)\right)=D\left(Q_{k}\left(D\left(f_{2(n-1)}(y)\right)\right)\right)
$$

$$
\begin{aligned}
g_{2(n-1)+k}(y) & =Q_{k}^{\prime}\left(D\left(f_{2(n-1)}(y)\right)\right) g_{2(n-1)}(y) \\
& =\frac{1}{\lambda} Q_{k}^{\prime}\left(D\left(f_{2(n-1)}(y)\right)\right) Q_{n-1}^{\prime}\left(\tilde{f}_{n-1}(y)\right) g_{n}(y) .
\end{aligned}
$$

Therefore,

$$
g_{3(n-1)}(y)=\frac{1}{\lambda} Q_{n-1}^{\prime}\left(D\left(f_{2(n-1)}(y)\right)\right) Q_{n-1}^{\prime}\left(\tilde{f}_{n-1}(y)\right) g_{n}(y) .
$$




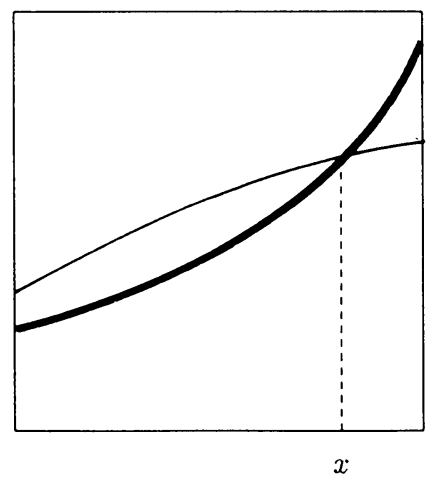

Figure 13. The map $\left.\left(D \circ f_{n-1}\right)\right|_{\Gamma_{A^{n-1}}}$ in Subcase (E1)

Iterating this process for $l=1,2, \ldots$ and for $y \in\left(x, x+\delta_{l}\right)$ we obtain

$$
g_{(l+2)(n-1)}(y)=\frac{1}{\lambda} g_{n}(y) Q_{n-1}^{\prime}\left(\tilde{f}_{n-1}(y)\right) \prod_{i=2}^{l+1} Q_{n-1}^{\prime}\left(D\left(f_{i(n-1)}(y)\right)\right) .
$$

Since $D\left(f_{k(n-1)}(x)\right)=c_{x}$ for all $l \geq 1$ and $\tilde{f}_{n-1}(x)=c_{x}$ we get

$$
g_{(l+2)(n-1)}(x)=\frac{1}{\lambda}\left(\dot{Q}_{n-1}^{\prime}\left(c_{x}\right)\right)^{l+1} g_{n}(x) \text {. }
$$

We recall that to end the proof of the lemma in Subcase (E1) we need to show that $g_{n}(x) \leq 0$. To see this we assume that $g_{n}(x)>0$ and we arrive to a contradiction. Since $s_{n-1}\left(\underline{A}^{n-1}\right) \in\{M, L\}$ and $D\left(f_{n-1}(x)\right)=c_{x}$, from the proof of Lemma 2.15 (see Figure 13), it follows that

$$
g_{n-1}(x)=Q_{n-1}^{\prime}\left(c_{x}\right) c_{x}^{\prime}>c_{x}^{\prime} \text {. }
$$

Hence $Q_{n-1}^{\prime}\left(c_{x}\right)>1$. Then, from $(\bullet)$ we obtain

$$
g_{(l+2)(n-1)}(x)>c_{x}^{\prime}
$$

for $l$ sufficiently large. Let $j \in \mathbf{N}$ be such that $(\star)$ holds with $l=j$. Set $\underline{A}^{(j+2)(n-1)}=\underline{A}^{n-1} \underline{A}_{+}^{n-1}\left(\underline{A}_{-}^{n-1}\right)^{j}$. Since $K(y)=\underline{A}^{(j+2)(n-1)}$ for all $y \in\left(x, x+\delta_{j}\right)$ we get that $\alpha_{\underline{A}^{(j+2)(n-1)}}=x$. Since the maps $D\left(f_{(j+2)(n-1)}(\mu)\right)$ and $c_{\mu}$ coincide at $\mu=x$ and $g_{(j+2)(n-1)}(x)>c_{x}^{\prime}$ it follows that $D\left(f_{(j+2)(n-1)}(y)\right)>c_{y}$ for $y \in\left(x, x+\delta_{j}\right)$ close enough to $x$. Thus, for such $y$ we have $K(y)=$ $\underline{A}^{(j+2)(n-1) R} d \cdots ;$ a contradiction with the fact that $K(y)>\underline{A}^{n-1} \underline{A}_{+}^{n-1}\left(\underline{A}_{-}^{n-1}\right)^{\infty}$. Then $g_{n}(x) \leq 0$ and hence $\left.f_{n}\right|_{\Gamma_{A^{n}}}$ is decreasing.

Subcase (E2). $s_{n-1}\left(\alpha_{\underline{A}^{n-1}}\right) \in\{C, R\}$. By the induction hypotheses we have that $c_{\alpha_{A^{n-1}}}$ is periodic of period $k<n-1$ and $K=\Gamma_{\underline{A}^{n-1}}$. Since $\left.f_{n}\right|_{\Gamma_{A^{n}}}$ is concave, to prove that $\left.f_{n}\right|_{\Gamma_{A^{n}}}$ is decreasing it suffices to show that $g_{n}\left(\alpha_{\underline{A}^{n-1}}\right) \leq$ 0 . Let $\underline{A}^{k}$ be the $n$-strip formed by the first $k$ symbols of $K\left(\alpha_{\underline{A}^{n-1}}\right)$ and set $Q_{j}\left(c_{\mu}\right)=f_{j}(\mu)$ for $j=1, \ldots, k$. Also set $n=k r+i$ with $k, r \in \mathbf{N}$ and $0<i \leq k$. As in the previous subcase we have $f_{n}(y)=Q_{i}\left(f_{r k}(y)\right)$ for $y$ close enough to $\alpha_{\underline{A}^{n-1}}$. Then, for such $y$, we obtain

$$
g_{n}(y)=Q_{i}^{\prime}\left(f_{r k}(y)\right) g_{r k}(y) \text {. }
$$


Since $f_{r k}\left(\alpha_{\underline{A}^{n-1}}\right)=c_{\alpha_{\underline{A}}^{n-1}}$ we have $g_{n}\left(\alpha_{\underline{A}^{n-1}}\right)=Q_{i}^{\prime}\left(c_{\alpha_{\underline{A}}{ }^{n-1}}\right) g_{r k}\left(\alpha_{\underline{A}^{n-1}}\right)$. We note that the signs of $Q_{i}^{\prime}\left(c_{\alpha_{A^{n-1}}}\right)$ and $g_{i}\left(c_{\alpha_{A^{n-1}}}\right)$ coincide and, by the induction hypotheses, this sign is equal to the parity of $\left(A_{0} \cdots A_{i-1}\right)$. Furthermore, the sign of $g_{r k}$ is equal to the parity of $\left(A_{0} \cdots A_{r k-1}\right)$ and hence, the sign of $g_{n}\left(\alpha_{\underline{A}^{n-1}}\right)$ is equal to the parity of $A_{0} \cdots A_{r k-1} A_{0} \cdots A_{i-1}=\underline{A}^{n}$. So $g_{n}\left(\alpha_{\underline{A}^{n-1}}\right) \leq 0$.

Lemma 2.23. Let $\mu_{1}, \mu_{2} \in I(m)$ be such that $\mu_{1}<\mu_{2}$ and $K\left(\mu_{1}\right) \neq K\left(\mu_{2}\right)$. Let $n$ be the first natural such that $A_{n}\left(\mu_{1}\right) \neq A_{n}\left(\mu_{2}\right)$. If $n>1$ then $s_{1}\left(\mu_{1}\right), \ldots$, $s_{n-1}\left(\mu_{1}\right) \in\{L, R\}$.

Proof. For $i=1, \ldots, n-1$ set $A_{i}={ }^{s_{i}} d_{i}=A_{i}\left(\mu_{1}\right)=A_{i}\left(\mu_{2}\right)$ and $\underline{A}^{i}=$ $A_{0} \cdots A_{i-1}$. Suppose that there exists $j \in\{1, \ldots, n-1\}$ such that $s_{j} \in\{M, C\}$ and let $0<k \leq n-1$ be the first natural with this property. By Lemma 2.22 we have that $\left.f_{k}\right|_{\Lambda_{A^{k}}}$ is a rational function which is increasing or decreasing according to the value of $\sigma\left(\underline{A}^{k}\right)$. Then, if $s_{k}=M$ we obtain that $\left.f_{k}\right|_{\left(\mu_{1}, \mu_{2}\right)}$ is constant; a contradiction. If $s_{k}=C$ we get $D\left(f_{k}(\mu)\right)=c_{\mu}$ for all $\mu \in\left(\mu_{1}, \mu_{2}\right)$. Hence $\left.f_{k}\right|_{\left[\mu_{1}, \mu_{2}\right]}$ is increasing. Also by Lemma 2.22, if $s_{i}=R$ for some $i=$ $1, \ldots, k-1$ we have that $\left.f_{k}\right|_{\left(\mu_{1}, \mu_{2}\right)}$ is convex. This gives a contradiction because the function $c_{\mu}$ is concave. Otherwise, by Lemma 2.18, we get that $f_{k}(\mu)=\lambda^{k} c_{\mu}+p(\lambda)$ where $p(\lambda)$ is a polynomial of degree $k-1$ in $\lambda$; again a contradiction with the fact that $D\left(f_{k}(\mu)\right)=c_{\mu}$.

Proposition 2.24. Let $\mu_{1}, \mu_{2} \in I(m)$ be such that $\mu_{1}<\mu_{2}$. Then $K\left(\mu_{1}\right) \leq$ $K\left(\mu_{2}\right)$.

Proof. Assume that $K\left(\mu_{1}\right) \neq K\left(\mu_{2}\right)$ and let $n$ be the first natural such that $A_{n}\left(\mu_{1}\right) \neq A_{n}\left(\mu_{2}\right)$. For $i=1, \ldots, n-1$ set $A_{i}=s_{i} d_{i}=A_{i}\left(\mu_{1}\right)=A_{i}\left(\mu_{2}\right)$ and $\underline{A}^{i}=A_{0} \cdots A_{i-1}$. By Lemma 2.23 we get $s_{i} \in\{L, R\}$ for $i=1, \ldots, n-1$.

Now, by Lemma 2.22 , we get that $\left.f_{n}\right|_{\left(\mu_{1}, \mu_{2}\right)}$ is strongly increasing or strongly decreasing according to the parity of $\sigma\left(\underline{A}^{n}\right)$. Thus we obtain $A_{n}\left(\mu_{1}\right) \leq A_{n}\left(\mu_{2}\right)$ if $\sigma\left(\underline{A}^{n}\right)=1$ or $A_{n}\left(\mu_{1}\right) \geq A_{n}\left(\mu_{2}\right)$ if $\sigma\left(\underline{A}^{n}\right)=-1$. In both cases we have $K\left(\mu_{1}\right) \leq K\left(\mu_{2}\right)$.

Now we are ready to prove Theorems 2.1 and 2.2.

Proof of Theorem 2.1. If $b\left(\mu_{1}\right) \neq b\left(\mu_{2}\right)$ the theorem holds from Proposition 2.3. So, we can assume that $\mu_{1}, \mu_{2} \in J(m)$. If $\lambda^{m-1} \mu<1$ then the map $\left.\left(F_{\mu}^{m}-1\right)\right|_{\left[\lambda^{m-1} /\left(\lambda^{m}-1\right), f_{m}(\mu)-1\right]}$ (see Proposition 2.13 and Figures 4 and 14) has a fixed point $z$ such that $\underline{I}_{F_{\mu}}(z)=\left({ }^{R} 1\left({ }^{L} 0\right)^{m-1}\right)^{\infty}$. Clearly we get $K(\mu)=$ ${ }^{C} 1\left({ }^{L} 0\right)^{m-1}\left({ }^{R} 1\left({ }^{L} 0\right)^{m-1}\right)^{\infty}$. Then, when $\lambda^{m-1} \mu<1$ the kneading sequence $K(\mu)$ remains constant.

Now assume that $\lambda^{m-1} \mu_{2}>1$. If additionally $\mu_{2} \leq \lambda /\left(\lambda^{m}-1\right)$ then, by Lemma 2.9, it follows that $\lambda>\pi_{m}$. From Proposition 2.13 we obtain $K\left(\mu_{1}\right)<$ $K\left(\mu_{2}\right)$. So we assume that $\mu_{2}>\lambda /\left(\lambda^{m}-1\right)$. If $\mu_{1}<\lambda /\left(\lambda^{m}-1\right)$ we obtain the desired result from Lemma 2.9. Lastly we assume that $\mu_{1}, \mu_{2} \in I(m)$. From Proposition 2.24 we get $K\left(\mu_{1}\right) \leq K\left(\mu_{2}\right)$. Suppose that $K\left(\mu_{1}\right)=K\left(\mu_{2}\right)$. Then we obtain $K(\mu)=K\left(\mu_{1}\right)$ for all $\mu \in\left[\mu_{1}, \mu_{2}\right]$. Set $K\left(\mu_{1}\right)=A_{0} A_{1} \cdots=$ $s_{0} d_{0}^{s_{1}} d_{1} \cdots$ and $\underline{A}^{n}=A_{0} \cdots A_{n-1}$. Then, from Lemma 2.23, we get $s_{i} \in\{L, R\}$ for all $i>0$. Since $\left[\mu_{1}, \mu_{2}\right] \subset \Gamma_{A^{n}}$ for all $n \geq 0,\left.f_{n}\right|_{\left[\mu_{1}, \mu_{2}\right]}$ is a rational 


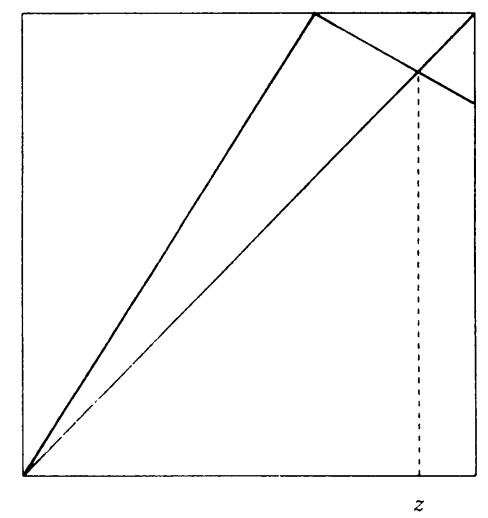

FIGURE 14. The map $\left.\left(F_{\mu}^{m}-1\right)\right|_{\left[\lambda^{m-1} /\left(\lambda^{m}-1\right), f_{m}(\mu)-1\right]}$ when $\lambda^{m-1} \mu<1$

function for all $n \geq 0$. Suppose that there exists $n$ such that $s_{n}=R$ and let $k$ be the first natural with the property $s_{k}=R$. From Lemma 2.18 we have

$$
g_{k}^{\prime}(\mu)=-2 \frac{\lambda^{k}(\lambda-1)}{(\mu+\lambda)^{3}}
$$

for all $\mu \in\left(\mu_{1}, \mu_{2}\right)$. On the other hand, by Lemma 2.22 , the map $\left.f_{n}\right|_{\left[\mu_{1}, \mu_{2}\right]}$ is either increasing and convex or decreasing and concave according to the parity of $\underline{A}^{n}$ for all $n>k$. In particular $g_{n}$ and $g_{n}^{\prime}$ have the same sign for all $n>k$. Now set $k_{1}=\min \left(\lambda^{m}, \lambda^{m-1} \mu_{1}\right)$ and $k_{2}=2 \lambda^{k}(\lambda-1) /\left(\mu_{2}+\lambda\right)^{3}$. Since $\mu_{1}>\lambda /\left(\lambda^{m}-1\right)$ we get $k_{1}>1$. We claim that

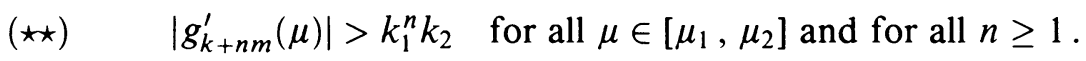

To see this note that for all $i>0$ either $g_{k+i m}^{\prime}(\mu)=\lambda^{m} g_{k+m(i-1)}^{\prime}(\mu)$ or $g_{k+i m}^{\prime}(\mu)=-2 \lambda^{m-1} g_{k+m(i-1)}(\mu)-\lambda^{m-1} \mu g_{k+i(m-1)}^{\prime}(\mu)$. Then, since $g_{k+(i-1) m}$ and $g_{k+(i-1) m}^{\prime}$ have the same sign we obtain $\left|g_{k+i m}^{\prime}\right|>k_{1}\left|g_{k+(i-1) m}^{\prime}\right|$ on $\left[\mu_{1}, \mu_{2}\right]$. From this $(\star \star)$ follows inductively.

Now fix $x \in\left(\mu_{1}, \mu_{2}\right)$ and let $n \geq 1$ be such that $k_{1}^{n} k_{2}>\frac{1}{\left(\mu_{2}-x\right)\left(x-\mu_{1}\right)}$. Then since $g_{k+n m}$ and $g_{k+n m}^{\prime}$ have the same sign and it is constant on $\left[\mu_{1}, \mu_{2}\right]$ we have

$$
\left|g_{k+n m}(x)\right|=\left|g_{k+n m}\left(\mu_{1}\right)\right|+\int_{\mu_{1}}^{x}\left|g_{k+n m}^{\prime}(\mu)\right| d \mu>\frac{1}{\mu_{2}-x} .
$$

Then, since $\left|g_{k+n m}\right|_{\left[\mu_{1}, \mu_{2}\right]}$ is increasing,

$$
\left|g_{k+n m}(\mu)\right|>\frac{1}{\mu_{2}-x} \text { for all } \mu \in\left[x, \mu_{2}\right] .
$$

From the mean value theorem we get

$$
\left|f_{k+n m}\left(\mu_{2}\right)-f_{k+n m}(x)\right|=\left|g_{k+n m}(\xi)\right|\left(\mu_{2}-x\right)
$$

for some $\xi \in\left(x, \mu_{2}\right)$ and hence $\left|f_{k+n m}\left(\mu_{2}\right)-f_{k+n m}(x)\right|>1$. On the other hand, by Lemma $2.17, E\left(f_{k+n m}(\mu)\right)$ is constant for all $\mu \in \Gamma_{\underline{A}^{k+n m}}$. Namely, $E\left(f_{k+n m}(\mu)\right)=\sum_{i=0}^{k+n m-1} d_{i}$. This contradicts the fact that $\left|f_{k+n m}\left(\mu_{2}\right)-f_{k+n m}(x)\right|$ $>1$. 
Now we consider the case $s_{i}=L$ for all $i>0$. From Lemma 2.18, $g_{i}(\mu)=$ $\lambda^{i} c_{\mu}^{\prime}$ for all $i>0$. Let $n$ be such that $\lambda^{n} c_{\mu_{2}}^{\prime}>\frac{1}{\mu_{2}-\mu_{1}}$. For such $n$ we have

$$
g_{n}(\mu)=\lambda^{n} c_{\mu}^{\prime} \geq \lambda^{n} c_{\mu_{2}}^{\prime}>\frac{1}{\mu_{2}-\mu_{1}}
$$

for all $\mu \in\left[\mu_{1}, \mu_{2}\right]$. Therefore, from the mean value theorem we obtain

$$
f_{n}\left(\mu_{2}\right)-f_{n}\left(\mu_{1}\right)=g_{n}(\xi)\left(\mu_{2}-\mu_{1}\right)
$$

for some $\xi \in\left[\mu_{1}, \mu_{2}\right]$ and hence $f_{n}\left(\mu_{2}\right)-f_{n}\left(\mu_{1}\right)>1$. As above we obtain a contradiction. This ends the proof of the theorem.

Proof of Theorem 2.2. First we consider the case $b\left(\mu_{1}\right), b\left(\mu_{2}\right) \in J(m)$. Assume that $\mu \leq \lambda /\left(\lambda^{m}-1\right)$ and set $F=F_{\mu}$. By Remark 2.10, we have that $F^{m}-1$ maps the interval

$$
J=\left[\frac{\lambda^{m-1}}{\lambda^{m}-1}, F^{m}\left(c_{\mu}\right)-1\right]
$$

into itself. Then the set of nonwandering points is the union of two invariant closed sets: one of them is contained in $Z=\bigcup_{k \in \mathbf{Z}}\left(k+\bigcup_{i=0}^{m-1} F^{i}(J)\right)$ and the other one in the closure of the complement of $Z$ (think about the situation on the circle). Then the entropy is equal to the maximum of the entropies on these two sets. By the unimodality of $\left.F^{m}\right|_{J}$, the entropy of $F$ on the first one is at most $\frac{1}{m} \log 2$. To compute the entropy on the second one we collapse each of the connected components of $Z$ to a point and we get a bimodal map $\widetilde{F}$ with $\widetilde{F}(0)=0$ and $\underline{I}_{\widetilde{F}}\left(c_{\widetilde{F}}\right)=\left({ }^{C} 1\left({ }^{L} 0\right)^{m-1}\right)^{\infty}$. Since $\widetilde{F}$ has the same kneading sequences as $F_{0,1 / m}^{-}$it follows that $h(\widetilde{F})=\log \beta_{0,1 / m}^{-}$(see [AM]). On the other hand, since $\mu \leq \lambda /\left(\lambda^{m}-1\right), L_{F}=\left[0, \frac{1}{m}\right]$. Hence $L_{F^{m}}=[0,1]$. So, by Theorem 1.2 of [ALMM], $s\left(F^{m}\right) \geq \beta_{0,1}^{-}$. An easy computation shows that $\beta_{0,1}^{-}=\sqrt{2}+1>2$. Thus, $s(F)>2^{1 / m}$ and, hence, $h(F)=\log \beta_{0,1 / m}^{-}$.

Now assume that $\mu>\lambda /\left(\lambda^{m}-1\right)$ and set $F=F_{\mu}, c=c_{\mu}$, and $\phi=\phi_{F}$ the map defined in $\S 2$ of $[\mathrm{AM}]$. First we show that $\phi$ is a conjugacy. To do this suppose that the opposite is true. Then since $\phi$ is nondecreasing there exists $J=\left[x_{1}, x_{2}\right] \subset[0,1]$ such that $\left.\phi\right|_{J}$ is constant. Then, from Lemma 2.7(3) of $[\mathrm{AM}], \Lambda(J)=0$. From Lemma 2.6 we know that $\mathscr{T}_{F}$ is dense in $\mathbf{R}$ and, by taking $F^{n}(J)$ instead of $J$, we can assume that either $0 \in J$ or $c \in J$. If $0 \in J$ then there exists $k$ such that $[0,1] \subset D\left(F^{k}(J)\right)$; a contradiction. Hence $c \in J$. Now assume that $J$ is maximal with the properties that $c \in J$ and $\Lambda(J)=0$. If for some $k, 0 \in D\left(F^{k}(J)\right)$, as above, we get a contradiction. Since $\mathscr{T}_{F}$ is dense in $[0,1]$ we obtain that $c \in D\left(F^{k}(J)\right)$ for some $k \geq 1$. Now assume that $k$ is the first natural larger than 0 such that $c \in D\left(F^{k}(J)\right)$. By the maximality of $J$ we have $D\left(F^{k}(J)\right) \subset J$. Since $\{0, c\} \cap D\left(F^{i}(J)\right)=\varnothing$ for $i=1, \ldots, k-1$, we get that $A_{i}(x)$ remains constant when $x$ varies in $J$ for $i=1, \ldots, k-1$. We denote this value by $A_{i}={ }^{s_{i}} d_{i}$. Set

$$
q=\operatorname{Card}\left\{i \in\{1, \ldots, k-1\}: s_{i}=R\right\} .
$$

We note that from Lemma 2.4 and Remark 2.5 we get $k \geq m$ and if $s_{i}=R$ for some $i=1, \ldots, k-1$ then $i<k-(m-1)$ and $s_{i+1}=\cdots=s_{i+m-1}=L$. Since $s_{1}=\cdots=s_{m-1}=L$ we have $q m+m \leq k$. Then, $\left.D\left(F^{k}\right)\right|_{J}$ is piecewise 
linear with two pieces. Therefore $\left.D\left(F^{k}\right)\right|_{\left[x_{1}, c\right]}$ has slope $\left(\lambda^{m-1} \mu\right)^{q} \lambda^{k-m q}$ and $\left.D\left(F^{k}\right)\right|_{\left[c, x_{2}\right]}$ has slope $\left(\lambda^{m-1} \mu\right)^{q+1} \lambda^{k-m q-m}$. This gives a contradiction because

$$
\frac{1}{\left(\lambda^{m-1} \mu\right)^{q} \lambda^{k-m q}}+\frac{1}{\left(\lambda^{m-1} \mu\right)^{q+1} \lambda^{k-m q-m}}<\frac{1}{\lambda^{m}}+\frac{1}{\lambda^{m-1} \mu}<1 .
$$

Hence, $F_{\mu}$ and $\widetilde{F}_{\mu}$ are conjugate and, by Proposition 3.12 of [AM], (recall that from Remark 2.7, Proposition 3.12 of [AM] works in this case) $K(\mu)=$ $\underline{I}_{\widetilde{F}_{\mu}}\left(c_{\widetilde{F}_{\mu}}\right)$. Therefore, if $\mu_{1}, \mu_{2} \in I(m)$ and $\mu_{1}<\mu_{2}$ then, from Theorem 2.1

we have that $K\left(\mu_{1}\right)<K\left(\mu_{2}\right)$ and hence $\widetilde{F}_{\mu_{1}} \neq \widetilde{F}_{\mu_{2}}$. Since $T\left(F_{\mu_{1}}\right)=T\left(F_{\mu_{2}}\right)=0$ we obtain $s\left(F_{\mu_{1}}\right) \neq s\left(F_{\mu_{2}}\right)$. On the other hand, since $K\left(\mu_{1}\right)<K\left(\mu_{2}\right)$, then $s\left(\mu_{1}\right) \leq s\left(\mu_{2}\right)$. So, $s\left(\mu_{1}\right)<s\left(\mu_{2}\right)$ and $h\left(\mu_{1}\right)<h\left(\mu_{2}\right)$. If $\mu_{2}>\lambda /(\lambda-1) \geq \mu_{1}$ take $\mu_{3} \in\left(\lambda /\left(\lambda^{m}-1\right), \mu_{2}\right)$. Then, by the above argument we have $h\left(\mu_{2}\right)>$ $h\left(\mu_{3}\right) \geq h\left(\mu_{1}\right)$. Hence $(2)$ holds.

Lastly, assume that $\mu_{1} \in J\left(m_{1}\right)$ and $\mu_{2} \in J\left(m_{2}\right)$ with $m_{1} \neq m_{2}$. By Proposition 2.3 we have $m_{1}>m_{2}$. Let $\mu_{3} \in I\left(m_{1}\right)$ be such that $\mu_{1}<\mu_{3}$. Then $h\left(\mu_{1}\right)<h\left(\mu_{3}\right)$. From Proposition 2.3 we have $K\left(\mu_{3}\right)<K\left(\mu_{2}\right)$ and hence $h\left(\mu_{3}\right) \leq h\left(\mu_{2}\right)$. So, $h\left(\mu_{1}\right)<h\left(\mu_{3}\right) \leq h\left(\mu_{2}\right)$.

\section{THE FAMILY $F_{\lambda}$}

In this section we assume that $\mu$ is fixed and we study the uniparametric family $F_{\lambda}=G_{\lambda, \mu}$. We use a similar notation to the preceding section. We set $c_{\lambda}=c_{\lambda, \mu}, b(\lambda)=b_{\lambda, \mu}, f_{n}(\lambda)=F_{i}^{n}\left(c_{\lambda}\right)$ and $g_{n}(\lambda)=d f_{n}(\lambda) / d \lambda$ when $D\left(f_{i}(\lambda)\right) \notin\left\{0, c_{\lambda}\right\}$ for $i=1, \ldots, n-1$. Also set $K(\lambda)=K(\lambda, \mu)=$ $A_{0}(\lambda) A_{1}(\lambda) \cdots={ }^{C} d_{0}(\lambda)^{s_{1}(\lambda)} d_{1}(\lambda) \cdots$, and define

$$
\sigma_{n}(\lambda)= \begin{cases}1 & \text { if } \operatorname{Card}\left\{i \in\{1, \ldots, n-1\}: s_{i}(\lambda)=R\right\} \text { is even, } \\ -1 & \text { if } \operatorname{Card}\left\{i \in\{1, \ldots, n-1\}: s_{i}(\lambda)=R\right\} \text { is odd. }\end{cases}
$$

Lastly, set $c_{\lambda}^{\prime}=d c_{\lambda} / d \lambda=-(\mu+1) /(\lambda+\mu)^{2}$.

The main results of this section are the following.

Theorem 3.1. Let $\lambda_{1}$ and $\lambda_{2}$ be such that $\lambda_{1}<\lambda_{2}$. Then $K\left(\lambda_{1}\right) \leq K\left(\lambda_{2}\right)$. Moreover $K\left(\lambda_{1}\right)=K\left(\lambda_{2}\right)$ if and only if there exists $m \in \mathbf{Z}, m \geq 1$ such that $b\left(\lambda_{1}\right)=b\left(\lambda_{2}\right)=\frac{1}{m}$ and $\lambda_{2}^{m-1} \mu<1$.

Theorem 3.2. Let $\lambda_{1}$ and $\lambda_{2}$ be such that $\lambda_{1}<\lambda_{2}$. Then $h\left(\lambda_{1}\right) \leq h\left(\lambda_{2}\right)$. Moreover $h\left(\lambda_{1}\right)=h\left(\lambda_{2}\right)$ if and only if there exists $m \in \mathbf{Z}, m \geq 1$ such that $b\left(\lambda_{1}\right)=b\left(\lambda_{2}\right)=\frac{1}{m}$ and $\mu \leq \lambda_{2} /\left(\lambda_{2}^{m}-1\right)$. In this case $h\left(\lambda_{1}\right)=\beta_{0,1 / m}^{-}$.

Theorems 3.1 and 3.2 will be proved in a similar way to Theorems 2.1 and 2.2.

The next result is the equivalent to Proposition 2.3 for this new family. Its proof is analogous.

Proposition 3.3. If $\lambda^{\prime}>\lambda$ and $b\left(\lambda^{\prime}\right) \neq b(\lambda)$ then $K\left(\lambda^{\prime}\right)>K(\lambda)$.

From Proposition 3.3 it suffices to prove Theorem 3.1 when $b\left(\lambda^{\prime}\right)=b(\lambda)$.

Let $m \in \mathbf{N}$ be such that $m-1<b(\lambda) \leq m$. Clearly if $b(\lambda) \geq 1$ we have $m=1$ and $b(\lambda) \in\left[\frac{1}{m}, \frac{1}{m-1}\right)$ otherwise. We shall keep this assumption until the end of this section and again we shall split the study of this case into several lemmas. 


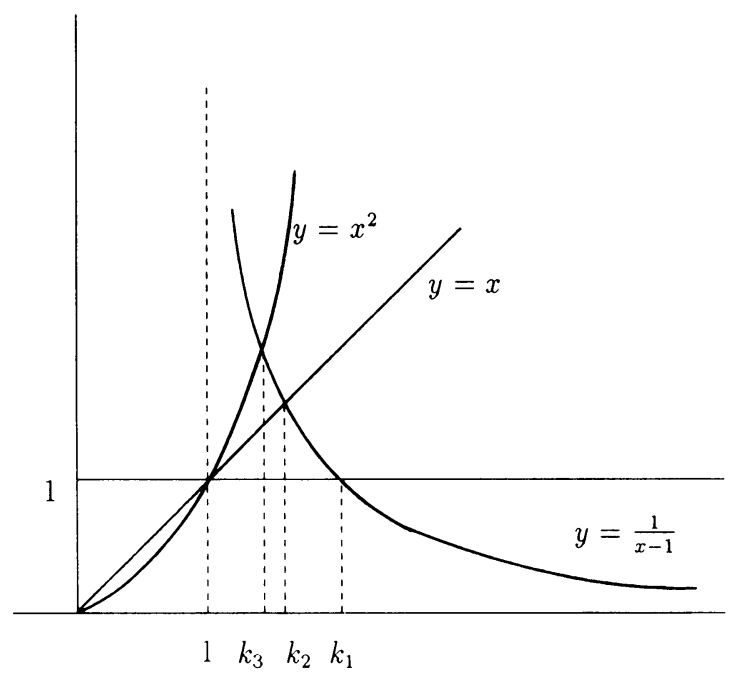

FIGURE 15. The roots $k_{m}$ of $R_{m}(\lambda)=0$

First we state results analogous to Lemmas 2.4 and 2.9 for the family $F_{\lambda}$. To do this we need some notation.

Set $R_{m}(\lambda)=\lambda^{m}-\lambda^{m-1}-1$. Since for $\lambda>1$ the equation $R_{m}(\lambda)=0$ is equivalent to $\lambda^{m-1}=1 /(\lambda-1), \lim _{x \rightarrow 1^{+}} 1 /(\lambda-1)=\infty, 1 /(\lambda-1)$ is decreasing and $\lambda^{m-1}$ is increasing (see Figure 15), we have that $R_{m}(\lambda)$ has a single root $k_{m}$ in $(1, \infty)$. Since for $\lambda \geq 2,1 /(\lambda-1) \leq 1$ we obtain $k_{m} \leq 2$ for all $m \geq 1$. Also, if $m>n$ we get $k_{m}<k_{n}$. Moreover, for $\lambda>k_{m}$ we have $R_{m}(\lambda)>0$.

Now we consider the maps $\varphi_{m}:\left(k_{m}, \infty\right) \rightarrow(0, \infty)$ and $\psi_{m}:(1, \infty) \rightarrow$ $(0, \infty)$ defined as follows:

$$
\varphi_{m}(\lambda)=\frac{1}{\lambda^{m}-\lambda^{m-1}-1} \quad \text { and } \quad \psi_{m}(\lambda)=\frac{\lambda}{\lambda^{m}-1} .
$$

Clearly we have that $\varphi_{m}$ and $\psi_{m}$ are decreasing, $\lim _{\lambda \rightarrow k_{m}^{+}} \varphi_{m}(\lambda)=\infty$, $\lim _{\lambda \rightarrow \infty} \varphi_{m}(\lambda)=0, \lim _{\lambda \rightarrow 1^{+}} \psi_{m}(\lambda)=\infty$, and

$$
\lim _{\lambda \rightarrow \infty} \psi_{m}(\lambda)= \begin{cases}0 & \text { if } m>1, \\ 1 & \text { if } m=1 .\end{cases}
$$

From Lemma 2.9 we get that $\varphi_{m}(\lambda)<\psi_{m}(\lambda)$ if and only if $\lambda>\pi_{m}$. Let $\varphi_{m}^{-1}:(0, \infty) \rightarrow\left(k_{m}, \infty\right)$ and $\psi_{m}^{-1}:(a, \infty) \rightarrow(0, \infty)$ be the inverse functions of $\varphi_{m}$ and $\psi_{m}$ respectively (where $a=1$ if $m=1$ and $a=0$ otherwise). Clearly $\varphi_{m}^{-1}$ and $\psi_{m}^{-1}$ are decreasing (see Figure 16). We note that when $m=1$ and $\mu \leq 1, \psi_{m}^{-1}(\mu)$ is not defined.

The next lemma is the analogue of Lemma 2.4 for the family $F_{\lambda}$.

Lemma 3.4. The following statements hold.

(1) If $m=1$ then $\lambda \in\left[\varphi_{m}^{-1}(\mu), \infty\right)$;

(2) If $m>1$ then $\lambda \in\left[\varphi_{m}^{-1}(\mu), \varphi_{m-1}^{-1}(\mu)\right)$;

(3) If $m>1$ then

$$
\left({ }^{C} 1\left({ }^{L} 0\right)^{m-1}\right)^{\infty}=K\left(\varphi_{m}^{-1}(\mu)\right) \leq K(\lambda)<\left({ }^{C} 1\left({ }^{L} 0\right)^{m-2}\right)^{\infty} .
$$

Otherwise $K(\lambda) \geq\left({ }^{C} 1\right)^{\infty}$. 


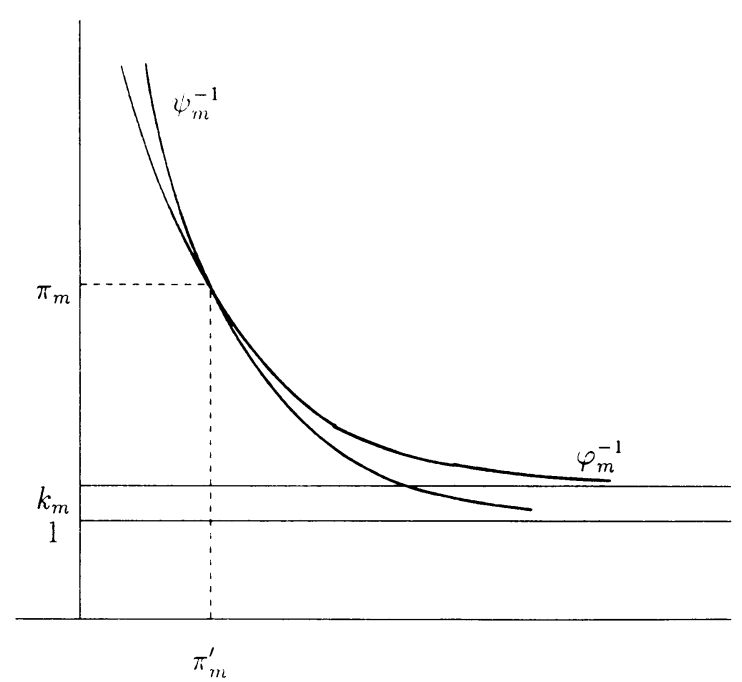

FIGURE 16. The maps $\varphi_{m}^{-1}$ and $\psi_{m}^{-1}$ for $m>1$

Proof. (3) follows in a similar way to Lemma 2.2(4). Also, from Lemma 2.4(1), we get $R_{m}(\lambda)>0$ and hence $\lambda>k_{m}$. Thus, by Lemma 2.4(2) it follows that $\varphi_{m}(\lambda) \leq \mu$. Hence $\lambda \geq \varphi_{m}^{-1}(\mu)$. This proves (1) and the first inequality of (2). Now assume that $m>1$. If $\lambda>k_{m-1}$ we get that $R_{m-1}(\lambda)>0$ and, by Lemma 2.4(3), it follows that $\mu<\varphi_{m-1}(\lambda)$. Thus, $\lambda<\varphi_{m-1}^{-1}(\mu)$. If $\lambda \leq k_{m-1}$ we have $\varphi_{m-1}^{-1}(\mu)>k_{m-1} \geq \lambda$.

Now we set

$$
\kappa^{\prime}(m)= \begin{cases}\varphi_{m-1}^{-1}(\mu) & \text { if } m>1, \\ \infty & \text { if } m=1,\end{cases}
$$

and $J_{m}^{\prime}=\left[\varphi_{m}^{-1}(\mu), \kappa^{\prime}(m)\right]$. Also set $\pi_{m}^{\prime}=\varphi_{m}\left(\pi_{m}\right)=\psi_{m}\left(\pi_{m}\right)$ and $I^{\prime}(m)=$ $\left\{\lambda \in J^{\prime}(m): \lambda>\psi_{m}^{-1}(\mu)\right\}$. When $m=1$ and $\mu \leq 1$ we set $I^{\prime}(m)=\varnothing$.

Remark 3.5. From Lemma 3.4(4) it follows that for $m>1$ and for $\lambda \in J^{\prime}(m)$ we have $K(\lambda)={ }^{C} 1\left({ }^{L} 0\right)^{m-2 L} d \cdots$ with $d \in\{0,1\}$. Therefore, from Proposition A of [AM], we get that if $d_{i}(\lambda)=1$ for some $i \geq 0$, then $s_{i+j}(\lambda)=L$ for $j=1, \ldots, m-1$ and $d_{i+j}(\lambda)=0$ for $j=1, \ldots, m-2$.

The following result is the analogue of Lemma 2.9 for the family $F_{\lambda}$ and follows easily from its proof. When $\lambda \in J^{\prime}(m)$ we define $x_{\lambda}$ as for the family $F_{\mu}$. That is, $x_{\lambda}$ is the $F_{\lambda}$-conjugate in $\left[c_{\lambda}, 1\right)$ of the largest element of the TPO of period $m$ and rotation number $\frac{1}{m}$ in $\left(0, c_{\lambda}\right]$ (recall that this TPO exists in view of Lemma 4.1 of $[\mathrm{AM}])$.

Lemma 3.6. The following statements hold.

(1) If $m>1$, or $m=1$ and $\mu>1$ then $\psi_{m}^{-1}(\mu) \in J^{\prime}(m)$ if and only if $\mu \leq \pi^{\prime}$

(2) If $m>1$, or $m=1$ and $\mu>1$ then for $\lambda \in J^{\prime}(m), f_{m}(\lambda) \leq 1+x_{\lambda}$ if and only if $\lambda \leq \psi_{m}^{-1}(\mu)$

(3) If $m=1$ and $\mu \leq 1$ then $f_{m}(\lambda) \leq 1+x_{\lambda}$ for all $\lambda \in J^{\prime}(1)$; 
(4) If $\mu \leq \pi^{\prime}$ and $\lambda, \lambda^{\prime} \in J^{\prime}(m)$ with $\lambda<\psi_{m}^{-1}(\mu)<\lambda^{\prime}$ then

$$
K(\lambda)<K\left(\psi_{m}^{-1}(\mu)\right)=\underline{B}<K\left(\lambda^{\prime}\right) ;
$$

(5) If $\mu>\pi_{m}^{\prime}$ and $\lambda \in J^{\prime}(m)$ then $K(\lambda)>\underline{B}$.

In view of Lemma 3.6 we can split our problem into two different cases. Namely $\mu<\pi_{m}^{\prime}$ and $\lambda \in\left[\varphi_{m}^{-1}(\mu), \psi_{m}^{-1}(\mu)\right]$ and

$$
\lambda \in\left(\max \left(\varphi_{m}^{-1}(\mu), \psi_{m}^{-1}(\mu)\right), \kappa^{\prime}(m)\right) .
$$

To study the monotonicity of $K(\lambda)$ in the first case we use again Theorem 2.6. The proof of the following result is similar to the proof of Proposition 2.13.

Proposition 3.7. Let $\mu, \lambda_{1}$, and $\lambda_{2}$ be such that $\mu<\pi_{m}^{\prime}$ and $\varphi_{m}^{-1}(\mu) \leq \lambda_{1}<$ $\lambda_{2} \leq \psi_{m}^{-1}(\mu)$. Then $K\left(\lambda_{1}\right) \leq K\left(\lambda_{2}\right)$. Moreover, if $\lambda_{2}^{m-1} \mu>1$ then $K\left(\lambda_{1}\right)<$ $K\left(\lambda_{2}\right)$.

Now, we consider the second case. That is

$$
\lambda \in I^{\prime}(m)=\left(\max \left(\varphi_{m}^{-1}(\mu), \psi_{m}^{-1}(\mu)\right), \kappa^{\prime}(m)\right) .
$$

We study this case through a sequence of lemmas.

Let $\underline{A}^{n}$ be an $n$-strip. Now we denote by $\Delta_{\underline{A}^{n}}$ the interior of the set of $\lambda$ 's such that $K(\lambda)$ starts with $\underline{A}^{n}$. We note that if $\Delta_{A^{n}} \neq \varnothing$ then $s_{0}=C$ and $\left.f_{n}\right|_{\Delta_{A^{n}}}$ is a rational function on $\lambda$. Now set $\Gamma_{\underline{A}^{n}}=\bar{\Delta}_{\underline{A}^{n}} \cap I^{\prime}(m)$.

Let $(a, b) \subset(1, \infty)$ and $f:(a, b) \rightarrow[0,1]$ be a continuous map. We say that $f$ is strongly increasing if $f$ is increasing and for all $\lambda, \lambda^{\prime} \in(a, b)$ with $\lambda<\lambda^{\prime}$ we have $A_{\lambda}(f(\lambda)) \leq A_{\lambda^{\prime}}\left(f\left(\lambda^{\prime}\right)\right.$ ) (now $A_{\lambda}(x)$ denotes the $F_{\lambda}$-address of $x$ ). We say that $f$ is strongly decreasing if $f$ is decreasing and for all $\lambda, \lambda^{\prime} \in(a, b)$ with $\lambda<\lambda^{\prime}$ we have $A_{\lambda}(f(\lambda)) \geq A_{\lambda^{\prime}}\left(f\left(\lambda^{\prime}\right)\right)$. Note that, contrarily to the situation for the family $F_{\mu}$, since each turning point of $F_{\lambda}$ depends on $\lambda$ in a nonincreasing way, to show that $f$ is strongly increasing it is enough to show that $f$ is increasing (see Figure 17(a)). We also note that the notions of strongly decreasing and decreasing are not equivalent (see Figure 17(b)).

The next results follow in a way similar to the corresponding results for the family $F_{\mu}$ (see Figure 18 on page 681 ).

Remark 3.8. From Lemma 2.15 it follows that if $f:[a, b) \rightarrow \mathbf{R}$ is a differentiable function decreasing and concave and $f(a)>c_{a}$ then $f$ is strongly decreasing. Moreover, to see that $f$ is strongly decreasing it suffices to show that $A_{y}(f(y)) \leq A_{a}(f(a))$ for all $y \in[a, b)$.

Lemma 3.9. Let $\underline{A}^{n}={ }^{C} d_{0}{ }^{R} d_{1} \ldots{ }^{R} d_{n-1}$ be an $n$-strip. Then for all $\lambda \in \Delta_{\underline{A}^{n}}$ and $k=1, \ldots, n$ we have

$$
f_{k}(\mu)=(-\mu)^{k-1} \lambda c_{\mu}+p_{\underline{A}^{n}}^{k}(\mu)
$$

where $p_{\underline{A}^{n}}^{k}(\mu)$ is a polynomial of degree $k-1$ in $\mu$ which depends only on $d_{0}, \ldots, d_{n-1}$.

Remark 3.10. From the preceding lemma we see that if $\underline{A}^{n}={ }^{C} d_{0}{ }^{R} d_{1} \ldots{ }^{R} d_{n-1}$ then for all $\mu \in \Delta_{\underline{A}^{n}}$ and for $k=1, \ldots, n$ we have

$$
g_{k}(\lambda)=(-1)^{k-1} \mu^{k}(\mu+1) /(\mu+\lambda)^{2} .
$$




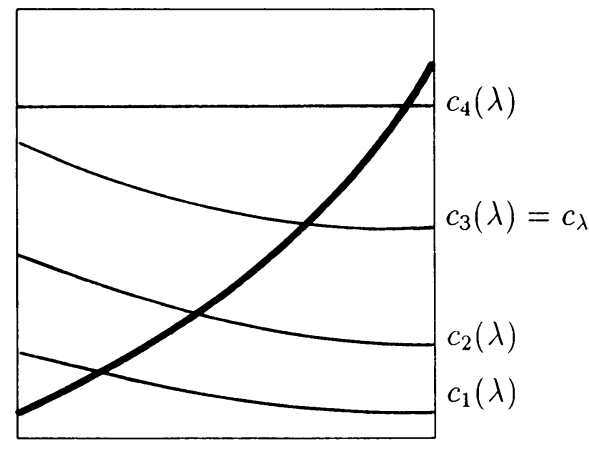

(a)

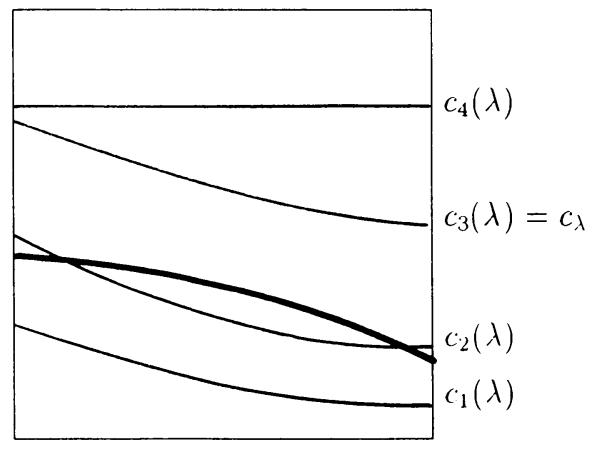

(b)

FigURE 17. (a) shows a map increasing and strongly increasing. (b) shows a map decreasing and not strongly decreasing. The curves $c_{i}(\mu)$ are the graphs of the turning points depending on $\lambda$

Moreover, if $\underline{C}^{k}$ is a $k$-strip and $\underline{A}^{n}=\underline{C}^{k} d_{k}{ }^{R} d_{k+1} \ldots{ }^{R} d_{n-1}$ then $g_{k+i}(\lambda)=$ $(-\mu)^{i} g_{k}(\lambda)$ for $i=1, \ldots, n-k$ and for $\lambda \in \Delta_{\underline{A}^{n}}$.

Lemma 3.11. Let $\underline{A}^{n}={ }^{s_{0}} d_{0} \ldots s_{n-1} d_{n-1}$ be an $n$-strip such that $s_{i} \in\{L, R\}$ for $i=1, \ldots, n-1$ and assume that $\Gamma_{\underline{A}^{n}}$ is an open interval, $\left.f_{n}\right|_{\Gamma_{A^{n}}}$ is either strongly increasing and $\sigma\left(\underline{A}^{n}\right)=1$ or strongly decreasing and $\sigma\left(\underline{A}^{n}\right)=-1$; and there exists a unique $x \in \Gamma_{\underline{A}^{n}}$ such that $D\left(f_{n}(x)\right)=c_{x}$. Then the following hold.

(1) If $\sigma\left(\underline{A}^{n}\right)=1$ then for all $\lambda \in \Gamma_{\underline{A}^{n}}$ such that $\lambda>x, K(\lambda)>\underline{A}^{n} \underline{A}_{+}^{n}\left(\underline{A}_{-}^{n}\right)^{\infty}$. Moreover, for all $l \in \mathbf{N}$ there exists $\delta_{l}$ such that $K(\lambda)=\underline{A}^{n} \underline{A}_{+}^{n}\left(\underline{A}_{-}^{n}\right)^{l} \ldots$ for all $\lambda \in\left(x, x+\delta_{l}\right)$.

(2) If $\sigma\left(\underline{A}^{n}\right)=-1$ then for all $\lambda \in \Gamma_{\underline{A}^{n}}$ such that $\lambda>x, K(\lambda)>\underline{A}^{n} \underline{A}_{-}^{n}\left(\underline{A}_{+}^{n}\right)^{\infty}$. Moreover, for all $l \in \mathbf{N}$ there exists $\delta_{l}$ such that $K(\lambda)=\underline{A}^{n} \underline{A}_{-}^{n}\left(\underline{A}_{+}^{n}\right)^{l} \cdots$ for all $\lambda \in\left(x, x+\delta_{l}\right)$.

Lemma 3.12. Let $\underline{A}^{n}=s_{0} d_{0} \ldots s_{n-1} d_{n-1}$ be an $n$-strip such that $s_{i} \in\{L, R\}$ for $i=1, \ldots, n-1$ and assume that $\Gamma_{A^{n}} \neq \varnothing$. Then the following hold.

(1) $\Gamma_{\underline{A}^{n}}=\left(\alpha_{\underline{A}^{n}}, \beta_{\underline{A}^{n}}\right)$ with $\beta_{\underline{A}^{n}} \in \mathbf{R} \cup\{\infty\}$.

(2) Either $\bar{D}\left(f_{n}\left(\alpha_{\underline{A}^{n}}\right)\right)=0$ or $c_{\alpha_{A^{n}}}$ is periodic of period less than $n$ or $\underline{A}^{n}=\underline{B}^{n}$ and in this case $\alpha_{\underline{A}^{n}}=\max \left(\varphi_{m}^{-1}(\mu), \psi_{m}^{-1}(\mu)\right)$.

(3) If $s_{i}=R$ for $i=1, \ldots, n-1$ then $\left.f_{n}\right|_{\Gamma_{A^{n}}}$ is strongly increasing if $\sigma\left(\underline{A}^{n}\right)=1$ and strongly decreasing if $\sigma\left(\underline{A}^{n}\right)=-1$. Otherwise, if $\sigma\left(\underline{A}^{n}\right)=1$ then $\left.f_{n}\right|_{\Gamma_{A^{n}}}$ is strongly increasing and convex and if $\sigma\left(\underline{A}^{n}\right)=-1$ then $\left.f_{n}\right|_{\Gamma_{A^{n}}}$ is strongly decreasing and concave.

Proof. The proof is very similar to the proof of Lemma 2.22 and it uses exactly the same ideas. Thus, rather than writing the whole proof we are going to point out its differences with the corresponding proof for the family $F_{\mu}$. So, from now on we shall use freely the whole proof of Lemma 2.22. We use induction. For $n=1$ we have $\underline{A}^{1}={ }^{C} d$ with $0<d \leq \widetilde{E}(\mu)+1$ (where $\widetilde{E}(x)$ denotes $E(x)$ for $x \in \mathbf{R} \backslash \mathbf{Z}$ and $x-1$ for $x \in \mathbf{Z})$. If $m>1$ then 


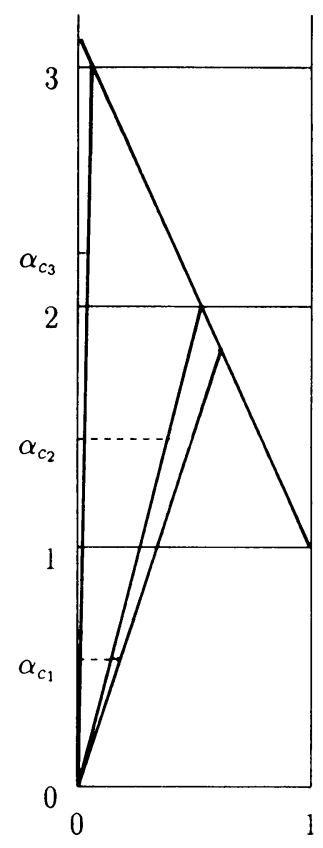

FIGURE 18. The values $\alpha_{C_{d}}$ for $d=1,2,3$

$\underline{A}^{1}=\underline{B}^{1}={ }^{C} 1$ and $\Gamma_{\underline{A}^{1}}=I^{\prime}(m)$. Hence (1) and (2) hold. If $m=1$ then $\Delta_{\underline{A}^{\prime}}=\left(d \mu /(\mu-d+1), \beta_{\underline{A}^{\prime}}\right)$ (see Figure 18) where

$$
\beta_{\underline{A}^{1}}= \begin{cases}\frac{(d+1) \mu}{\mu-d} & \text { if } d \leq \widetilde{E}(\mu), \\ \infty & \text { if } d>\widetilde{E}(\mu) .\end{cases}
$$

If $d=1$ then either $\mu \leq 1$ and $I^{\prime}(1)=\varnothing$ or $\mu>1$ and then we get $\underline{A}^{1}=\underline{B}^{1}$ and $\alpha_{A^{\prime}}=\max \left(\varphi_{m}^{-1}(\mu), \psi_{m}^{-1}(\mu)\right)$. If $d>1$, we have $\alpha_{\underline{A}^{\prime}}=(d-1) \lambda /(\lambda-\bar{d})$. Hence (1) and (2) hold for $n=1$.

Note that $f_{1}(\lambda)=\lambda c_{\lambda}$. Therefore $g_{1}(\lambda)=\mu(\mu+1) /(\mu+\lambda)^{2}>0$. Thus $\left.f_{1}\right|_{\Gamma_{A} 1}$ is increasing and hence it is strongly increasing.

Now we assume that the lemma holds for $n-1 \geq 1$ and prove it for $n$. The proof that (1) and (2) holds for $n$ is analogous to the corresponding proof of Lemma 2.22. To prove (3) we distinguish three cases.

Case $\left(\mathrm{A}^{\prime}\right) . s_{i}=R$ for $i=1, \ldots, n-2$. If $m>1$ then, by Lemma 3.4, we have $n=2$ and $\underline{A}^{2}={ }^{C} 1^{L} d$. Hence

$$
f_{2}(\lambda)=\lambda\left(f_{1}(\lambda)-1\right)+1 \quad \text { and } g_{2}(\lambda)=\lambda g_{1}(\lambda)+f_{1}(\lambda)-1>0,
$$

for all $\lambda \in \Gamma_{\underline{A}^{2}}$. Thus, $\left.f_{2}\right|_{\Gamma_{A^{2}}}$ is increasing and hence it is strongly increasing. Moreover, we get

$$
g_{2}^{\prime}(\lambda)=\lambda g_{1}^{\prime}(\lambda)+2 g_{1}(\lambda)=\frac{2 \mu(\mu+1)}{(\mu+\lambda)^{2}}-\frac{2 \lambda \mu(\mu+1)}{(\mu+\lambda)^{3}}=\frac{2 \mu^{2}(\mu+1)}{(\mu+\lambda)^{3}}>0
$$

for all $\lambda \in \Gamma_{\underline{A}^{2}}$. Then $\left.f_{2}\right|_{\Gamma_{A^{2}}}$ is convex. 
Now we consider the case $m=1$. If $d_{0}=1$ then $n \leq 3$ by Lemma 3.6(5). If $n=2$ then $\underline{A}^{2}=\underline{B}^{2}={ }^{C} 1^{R} 1$ and hence $g_{2}(\mu)=-\mu g_{1}(\mu)$. Thus $\left.f_{2}\right|_{\Gamma_{A^{2}}}$ is decreasing. Moreover,

$$
A_{2}\left(\alpha_{\underline{A}^{2}}\right)= \begin{cases}{ }^{C} 1 & \text { if } \alpha_{\underline{A}^{2}}=\varphi_{m}^{-1}(\mu), \\ { }^{L} 1 & \text { if } \alpha_{\underline{A}^{2}}=\psi_{m}^{-1}(\mu),\end{cases}
$$

because $\alpha_{\underline{A}^{2}}=\max \left(\varphi_{m}^{-1}(\mu), \psi_{m}^{-1}(\mu)\right)$. By Lemma 3.6(5) we obtain $A_{2}(\lambda) \leq{ }^{L} 1$ for all $\lambda \in \Gamma_{\underline{A}^{2}}$. Thus $\left.f_{2}\right|_{\Gamma_{\underline{A}^{2}}}$ is strongly decreasing. If $n=3$ we get $\underline{A}^{3}=$ ${ }^{C} 1^{R} 1^{L} d$ by Lemma 3.6. Since $s_{2}(\mu)=L$ for all $\lambda \in \Gamma_{\underline{A}^{2}}$ we have

$$
g_{3}(\lambda)=\lambda g_{2}(\lambda)+D\left(f_{2}(\lambda)\right) \text { and } g_{3}^{\prime}(\lambda)=2 g_{2}(\lambda)+\lambda g_{2}^{\prime}(\lambda)
$$

for all $\lambda \in \Gamma_{\underline{A}^{2}}$. An easy computation shows that

$$
g_{3}^{\prime}(\lambda)=-\frac{2 \mu^{3}(\mu+1)}{(\mu+\lambda)^{3}}<0
$$

for all $\lambda \in \Gamma_{\underline{A}^{2}}$. Since $\Gamma_{\underline{A}^{3}} \subset \Gamma_{\underline{A}^{2}}$ it follows that $\left.f_{3}\right|_{\Gamma_{A^{3}}}$ is concave. Then, to prove that $\left.f_{3}\right|_{\Gamma_{\underline{A}^{3}}}$ is decreasing it suffices to show that $g_{3}\left(\alpha_{\underline{A}^{2}}\right)<0$. To do this we use the same arguments as in Case (A) of Lemma 2.22 when $d_{0}=1$. Note that these arguments also show that $\left.f_{3}\right|_{\Gamma_{A^{3}}}$ is strongly decreasing.

Lastly, it remains to consider the case $d_{0}>1$. If $s_{n-1}=R$ we get $\left.g_{n}\right|_{\Gamma_{\underline{A}^{n}}}=$ $-\mu g_{n-1} \mid \Gamma_{\Lambda^{n}}$. Hence, $f_{n}$ is increasing or decreasing according to the parity of $\underline{A}^{n}$. Assume that $\sigma\left(\underline{A}^{n}\right)=-1$. Since $\underline{A}^{n} \neq \underline{B}^{n}$ (recall that $d_{0}>1$ ) we get that either $D\left(f_{n}\left(\alpha_{\underline{A}^{n}}\right)\right)=0$ or $\alpha_{\underline{A}^{n}}$ is periodic of period less than $n$. In the first case, by Remark 3.8, we obtain that $\left.f_{n}\right|_{\Gamma_{A}{ }^{n}}$ is strongly decreasing. In the second case we use the same arguments as in Case (B) of Lemma 2.22 to show that $\left.f_{n}\right|_{\underline{A}^{n}}$ is strongly decreasing. If $s_{n-1}=L$ we get from Remark 3.10

$$
g_{n-1}(\lambda)=(-1)^{n-2} \mu^{n-2} \frac{\mu(\mu+1)}{(\mu+\lambda)^{2}}
$$

and

$$
g_{n-1}^{\prime}(\lambda)=-2(-1)^{n-2} \mu^{n-2} \frac{\mu(\mu+1)}{(\mu+\lambda)^{3}}
$$

for all $\lambda \in \Gamma_{\underline{A}^{n-1}}$. Since $g_{n}(\lambda)=D\left(f_{n}(\lambda)\right)+\lambda g_{n-1}(\lambda)$ and $g_{n}^{\prime}(\lambda)=\lambda g_{n-1}^{\prime}(\lambda)+$ $2 g_{n-1}(\lambda)$ for all $\lambda \in \Gamma_{\underline{A}^{n}}$, we obtain

$$
\begin{aligned}
g_{n}^{\prime}(\lambda) & =-2(-1)^{n-2} \mu^{n-2} \frac{\lambda \mu(\mu+1)}{(\mu+\lambda)^{3}}+2(-1)^{n-2} \mu^{n-2} \frac{\mu(\mu+1)}{(\mu+\lambda)^{2}} \\
& =2(-1)^{n-2} \mu^{n-2} \frac{\mu(\mu+1)}{(\mu+\lambda)^{2}}\left(\frac{-\lambda}{\mu+\lambda}+1\right) \\
& =2(-1)^{n-2} \mu^{n-2} \frac{\mu^{2}(\mu+1)}{(\mu+\lambda)^{3}}
\end{aligned}
$$

for all $\lambda \in \Gamma_{\underline{A}^{n}}$. Hence, if $\sigma\left(\underline{A}^{n}\right)=1$ then $\left.f_{n}\right|_{\Gamma_{A^{n}}}$ is convex and if $\sigma\left(\underline{A}^{n}\right)=-1$, $\left.f_{n}\right|_{\Gamma_{\underline{A}^{n}}}$ is concave. If $\sigma\left(\underline{A}^{n}\right)=1$, from the induction hypotheses we get that 
$\left.f_{n-1}\right|_{\Gamma_{A^{n-1}}}$ is increasing. Since $g_{n}(\lambda)=D\left(f_{n}(\lambda)\right)+\lambda g_{n-1}(\lambda)$ for all $\lambda \in \Gamma_{\underline{A}^{n}}$ it follows that $\left.f_{n}\right|_{\Gamma_{A^{n}}}$ is increasing and hence it is strongly increasing. Assume that $\sigma\left(\underline{A}^{n}\right)=-1$. Since $\left.f_{n}\right|_{\Gamma_{A^{n}}}$ is concave, to see that $\left.f_{n}\right|_{\Gamma_{\Lambda^{n}}}$ is decreasing it suffices to show that $g_{n}\left(\alpha_{\underline{A}^{n}}\right) \leq 0$. To do this we use the same arguments of the proof of Case (E) of Lemma 2.22. To show that $\left.f_{n}\right|_{\Gamma_{A}{ }^{n}}$ is strongly decreasing we use either Remark 3.8 or the arguments of Case (B) of Lemma 2.22 depending on whether $D\left(\alpha_{\underline{A}^{n}}\right)=0$ or whether $\alpha_{\underline{A}^{n}}$ is periodic of period less than $n$.

Case $\left(\mathrm{B}^{\prime}\right) . \sigma\left(\underline{A}^{n-1}\right)=-1$ and $s_{j}=L$ for some $j \in\{1, \ldots, n-2\}$. Since $\sigma\left(\underline{A}^{n-1}\right)=-1$, by the induction hypotheses we know that $\left.f_{n-1}\right|_{\Gamma_{A^{n-1}}}$ is strongly decreasing and concave. If $s_{n-1}=R$ we get

$$
g_{n}(\lambda)=-\mu g_{n-1}(\lambda) \text { and } g_{n-1}^{\prime}(\lambda)=-\mu g_{n-1}(\lambda)
$$

for all $\lambda \in \Gamma_{\underline{A}^{n}}$. Hence $\left.f_{n}\right|_{\Gamma_{\underline{A}^{n}}}$ is strongly increasing and convex. If $s_{n-1}=R$ then

$$
g_{n}(\lambda)=D\left(f_{n-1}(\lambda)\right)+\lambda g_{n-1}(\lambda) \text { and } g_{n}^{\prime}(\lambda)=2 g_{n-1}(\lambda)+\lambda g_{n-1}^{\prime}(\lambda)
$$

for all $\lambda \in \Gamma_{\underline{A}^{n}}$. Therefore $\left.f_{n}\right|_{\Gamma_{A^{n}}}$ is concave. Then, to see that $\left.f_{n}\right|_{\Gamma_{A^{n}}}$ is decreasing it suffices to show that $g_{n}\left(\alpha_{\underline{A}^{n}}\right) \leq 0$. To do this we use the same arguments as in Case (E) of the proof of Lemma 2.22. To show that it is strongly decreasing we use the arguments of Case (B) of Lemma 2.22. In the particular case when $\underline{A}^{n}=\underline{B}^{n}$ we use again the corresponding arguments from the proof of Lemma 2.22 .

Case $\left(\mathrm{C}^{\prime}\right) . \quad \sigma\left(\underline{A}^{n-1}\right)=1$ and $s_{j}=L$ for some $j \in\{1, \ldots, n-2\}$. Since $\sigma\left(\underline{A}^{n-1}\right)=-1$, by the induction hypotheses we get that $\left.f_{n-1}\right|_{\Gamma_{A^{n-1}}}$ is strongly increasing and convex. If $s_{n-1}=L$ we have

$$
g_{n}(\lambda)=D\left(f_{n-1}(\lambda)\right)+g_{n-1}(\lambda)>0
$$

and

$$
g_{n}^{\prime}(\lambda)=2 g_{n-1}(\lambda)+\lambda g_{n-1}^{\prime}(\lambda)>0
$$

for all $\lambda \in \Gamma_{\underline{A}^{n}}$. Hence (3) holds in this case. If $s_{n-1}=R$ we have

$$
g_{n}(\lambda)=-\mu g_{n-1}(\lambda)<0 \quad \text { and } \quad g_{n}^{\prime}(\lambda)=-\mu g_{n-1}^{\prime}(\lambda)<0
$$

for all $\lambda \in \Gamma_{\underline{A}^{n}}$. Therefore $\left.f_{n}\right|_{\Gamma_{A^{n}}}$ is decreasing and concave. If $D\left(f_{n}\left(\alpha_{\underline{A}^{n}}\right)\right)=$ 0 , from Remark 3.8, we get that $\left.f_{n}\right|_{\Gamma_{A^{n}}}$ is strongly decreasing. If $\alpha_{\underline{A}^{n}}$ is periodic of period less than $n$, by using the arguments of Case (B) of Lemma 2.22, we obtain that $\left.f_{n}\right|_{\Gamma_{A^{n}}}$ is strongly decreasing. In the particular case when $\underline{A}^{n}={ }^{C} 1\left({ }^{L} 0\right)^{m-1 R} 1$ we use the corresponding arguments from Lemma 2.22.

The following proposition is the equivalent to Proposition 2.24 for the family $F_{\lambda}$. Its proof is analogous.

Proposition 3.13. Let $\lambda_{1}, \lambda_{2} \in I^{\prime}(m)$ be such that $\lambda_{1}<\lambda_{2}$. Then $K\left(\lambda_{1}\right) \leq$ $K\left(\lambda_{2}\right)$.

From all these results we can prove Theorems 3.1 and 3.2 in a similar way to Theorems 2.1 and 2.2 . 


\section{REFERENCES}

[ALMM] Ll. Alsedà, J. Llibre, F. Mañosas, and M. Misiurewicz, Lower bounds of the topological entropy for continuous maps of the circle of degree one, Nonlinearity 1 (1988), 463-479.

[ALMS] Ll. Alsedà, J. Llibre, M. Misiurewicz, and C. Simó, Twist periodic orbits and topological entropy of continuous maps of the circle of degree one which have a fixed point, Ergodic Theory Dynamical Systems 5 (1985), 501-517.

[AM] Ll. Alsedà and F. Mañosas, Kneading theory and rotation intervals for a class of circle maps of degree one, Nonlinearity 3 (1990), 413-452.

[BMT] K. Brucks, M. Misiurewicz, and Ch. Tresser, Monotonicity properties of the family of trapezoidal maps, Comm. Math. Phys. 137 (1991), 1-12.

[DH] A. Douady and J. H. Hubbard, Etude dynamique des polynomes complexes I and II, Publ. Math. Orsay, 84-02 (1984) and 85-04 (1985).

[MT] J. Milnor and W. Thurston, On iterated maps of interval. I, II, Lecture Notes in Math., vol. 1342, Springer-Verlag, Berlin and New York, 1988, pp. 465-563.

[M] M. Misiurewicz, Periodic points of maps of degree one of a circle, Ergodic Theory Dynamical Systems 2 (1982), 221-227.

[MV] M. Misiurewicz and E. Visinescu, Kneading sequences of skew tent maps, Ann. Inst. H. Poincaré Probab. Stat. 27 (1991), 125-140.

Departament de Matemàtiques, Facultat de Ciències, Universitat Autònoma de Barcelona, 08193 Bellaterra, Barcelona, Spain

E-mail address: imato@ec.uab.es

Departament de Matemàtica Aplicada II, E.T.S d'Enginyers Industrials de Terrassa, Universitat Politècnica de Catalunya, Colon 11, 08222 Terrasa, Barcelona, Spain 